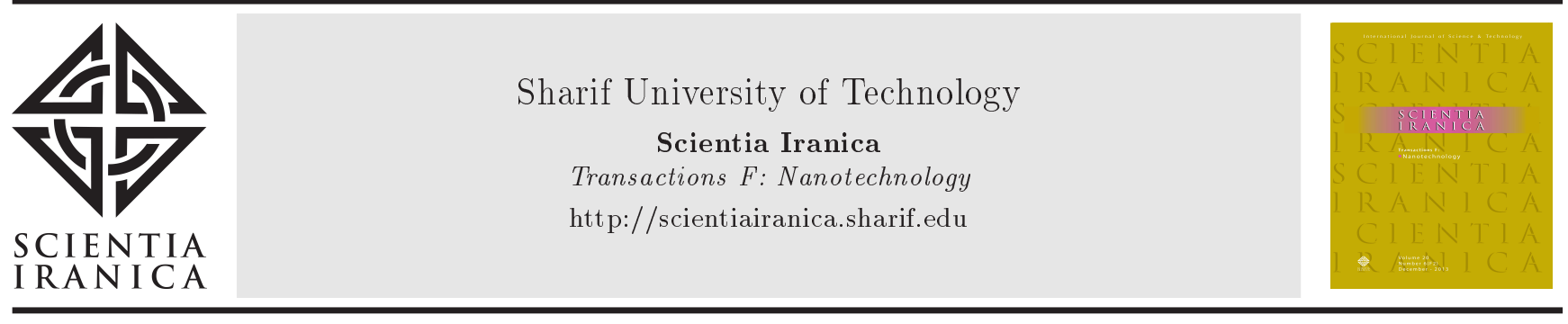

\title{
Transport of MHD nanofluid in a stratified medium containing gyrotactic microorganisms due to a stretching sheet
}

\author{
F. Shahzad*, M. Sagheer, and S. Hussain \\ Department of Mathematics, Capital University of Science and Technology (CUST), Islamabad, 44000, Pakistan.
}

Received 22 July 2020; received in revised form 17 June 2021; accepted 2 August 2021

\author{
KEYWORDS \\ Bioconvection; \\ Jeffrey nanofluid; \\ MHD; \\ Gyrotactic micro- \\ organisms; \\ Keller box method; \\ Stretching sheet; \\ Viscous dissipation.
}

\begin{abstract}
The present study aims to present an elaborated evaluation of stratified magnetohydrodynamics (MHD) Jeffrey nanofluid flow towards a stretching surface in the presence of gyrotactic micro-organisms. The flow was analyzed by considering viscous dissipation, Brownian motion, Joule heating, and thermophoresis as well as assuming the thermal, solutal, and motile density stratification aspects. The governing nonlinear system of Partial Differential Equations (PDEs) that controls the flow was turned into a group of nonlinear Ordinary Differential Equations (ODEs) using appropriate similarity transformation and later, they were numerically solved through Keller-box approach and algebraic software Matlab. The effects of different parameters on the fluid motion, heat, mass, density of the motile micro-organisms, local skin friction, local Nusselt number, local Sherwood number, and local density number of the motile micro-organisms were also evaluated using graphs and tables. The results indicated that the density of motile micro-organisms was a decreasing function of the bioconvection Lewis number, bioconvection Pecket number, and microorganisms' concentration difference. The Sherwood number and density rate of motile micro-organisms were higher in the case of magnetic parameter and Lewis number.

(C) 2021 Sharif University of Technology. All rights reserved.
\end{abstract}

\section{Introduction}

Bioconvection has received significant attention due to its extensive use cases as in the microbial fuel cell, modeling soil, gas-bearings sedimentary basin, microbial upgraded petroleum recovery biological systems, bioconvection nanotechnological devices, and biotechnology [1-3]. Bioconvection is a phenomenon that occurs when microorganisms that are denser than water run upward during the flow. As a result of this upward swimming, the micro-organisms are inclined to accumulate on the upper part of the fluids layer, thus

*. Corresponding author. Tel.: +92336-5583453 E-mail address: faisalshahzad309@yahoo.com (F. Shahzad)

doi: $10.24200 /$ sci.2021.56459.4734 leading to greater density stratification that usually becomes unstable. Swimming of these self-propelled motile micro-organisms generates enhanced density values that initiate bioconvection. Khan and Makinde [4] examined the magneto-hydrodynamics nanofluid containing gyrotactic micro-organisms past as stretching sheet. Of note, both stretching parameter and bioconvection Lewis number reduced the concentration of motile micro-organisms. Makinde and Animasaun [5] analyzed bioconvection in magneto-hydrodynamics nanofluid flow towards an upper sheet of a paraboloid of revolution by considering chemical reaction and nonlinear thermal emission. According to their observations, the local heat transport rate became closer to the decreasing values of temperature and larger values of Prandtl number. Xun et al. [6] evaluated the effect of thermal conductivity as well as temperature-dependent 
viscosity on the bio-convection flow in a rotating system and found that an increase in the thermal conductivity and viscosity would reduce the mass, heat transfer, and motile micro-organisms' flux on the surface. Mosayebidorcheh et al. [7] examined the effect of nano-bioconvection flow carrying nanoparticles as well as gyrotactic micro-organisms in a horizontal channel using modified least squares technique and found that the thermophoresis number had a negligible effect on the distribution of temperature profile and a strong impact on concentration and motile micro-organism.

Maxwell [8] attempted to enhance the thermals conductivity of a fluid by incorporating the micro-sized metallics particles into different base fluids; however, it increased erosion, additional flow resistance, and pressure loss. Choi and Eastman [9] proposed the idea of nanofluids to overcome the shortcomings of Maxwelltype fluids.

The name nanofluid was first introduced by Choi and Eastman [9] who formed these fluids by spreading nanoparticles in conventional base fluids to control heat transfer phenomenon. The suspended nanoparticles were composed of carbon allotropes, e.g., Carbon Nanotubes (CNTs), fullerene and metals, e.g., Au, $\mathrm{Cu}$, metal nitrides, e.g., AlN,SiN, oxide ceramics, e.g., $\mathrm{PbO}, \mathrm{CuO}$, and carbide ceramics, e.g., $\mathrm{CaC}_{2}$, Tic. Exploration of nanofluids has gained tremendous scientific significance due to a number of potential applications in a variety of areas including drugs and medications, manufacturing and materials, electronics, renewable energies, surfactant, transportation, environment, etc. [10,11]. Buongiorno [12] proposed a mathematical model that integrated the thermophoresis and Brownian motion effects in concentration with energy equations. Then, a number of authors including Sheikholeslami et al. [13], Shahrestani et al. [14], Lahmar et al. [15], Sheikholeslami and Farshad [16], and Ibrahim et al. [17] investigated the nanofluids to enhance the thermal conductivity. Sheikholeslami et al. [18] analytically investigated the heat transfer flow of a nanofluid and found that the temperature dynamics of nanofluids was affected by the concentration, shape, size, and material of the particles.

The mathematical models proposed for the motion of incompressible Newtonian fluids have drawn a number of mathematicians' attention over time. However, numerous fluid models found in the industry possess a rheology, not similar to that of Newtonian fluids, which includes shampoos, drilling mud, maizena, colloidal solutions, paint, alloys, grease, and sauce among others. Recently, non-Newtonians fluids have drawn remarkable attention due to their numerous applications in geophysics, extraction of crude soil from petroleum, soil sciences, ground water hydrology, spin coating, oceanography, astrophysics, polymer industry, engineering, etc. However, the constitutive relation of such fluids is more complex than that of Newtonian fluids. Consequently, the governing equations for non-Newtonian fluids are more complex than Navier-Stokes equations. For instance, Jeffreys model [19] is an effective and useful nonNewtonian fluid model which is time-derivative rather than convective-derivative. Jeffreys fluid is a rate fluid type, exhibiting the behavior of both relaxation and retardations time. Saif et al. [20], Narayana et al. [21], Jena et al. [22], Sreelakshmi et al. [23], and Ahmad et al. [24] have contributed to this area.

Stratification occurs as a result of differences in temperature, concentrations variations, and even existence of different fluid densities. This sort of phenomenon has gained significance as a result of its presence in the flows in rivers, lakes and seas, water reservoirs, etc. In addition, the effects of solute and thermal stratifications are of significance for solar power, mainly because better stratification is indicative of better energy productivity. Bearon and Grunbaum [25] examined the effect of bioconvection in a stratified environment. They concluded that as a result of reduction in the gyrotactic parameter, both velocity profile and cell concentration increased. Kameswaran et al. [26] examined the convective heat transfer in a thermally stratified nanofluid flow on a vertical wavy surface and found that the thermal stratification parameter could increase the thickness of the thermal boundary layer. Madhu and Reddy [27] evaluated the effect of thermal stratification on the magnetohydrodynamic heat transfer flow past an exponentially stretching surface and concluded that the temperature gradient was substantially enhanced with a boost in stratification.

The problems of heat transfer flow caused by stretching sheets have numerous industrial applications such as production of polythene, surgical equipment, fiber technology, metallurgical processes, glass blowing, manufacturing of synthetic sheets, hot rolling, and extrusion process, thus catching the attentions of a number of researchers for the last couple of decades. In the course of the process of extrusion, the high quality of the final product is expected by setting an appropriate speed of stretching along with the simultaneous cooling or heating throughout the procedure. Consequently, the characteristics of the heat transfer flow of a nanofluid past a stretching sheet have practical utility in a number of industrial as well as engineering processes. Sakiadis [28] developed the idea of hydrodynamic boundary layer induced by fluid flow towards a continuous solid sheet and determined the governing differential and integral equations of motion for these types of surfaces. Crane [29] examined the flow past a stretching sheet with a stretching speed related linearly to the horizontal distance from the slit. Tsou et al. [30] presented an experimental and 
analytical review of the flow and heat transportation characteristics in the boundary layer over a continuous moving plate. Then, a number of researchers have broadened the notion of stretching surface to a variety of fluid models [31-34].

The analysis of MHD flows has been found to be effective in several metallurgical, engineering, and industrial fields. Magnetohydrodynamics enjoys several notable applications in biomechanics, petroleum technologies, plasma studies, flow of blood measurements, MHD generators, etc. Sarpakaya [35] is regarded as the pioneer of this idea who studied the characteristics of magnetohydrodynamics flows of non-Newtonian fluid using an analytical method. Elbashbeshy et al. [36] examined the effects of thermal radiation and MHD on the time-dependents mixed convections flow past an exponentially extended surface including heat generation/absorption. Animasaun [37] referred to the effects of the variables viscosity, thermals conductivity, and thermophoresis on the magnetohydrodynamic flow of a Casson fluid with the consideration of the chemical reaction of the $n$th order. Nayak et al. [38] examined the effect of transverse magnetic fields on the 3D free convective flow of nanofluid towards a stretching surface. The irreversible viscous dissipation process is defined as the process in which the kinetic energy is transformed into the internal energy of fluid caused by its viscosity. Abel et al. [39] pointed to the impacts of buoyancy, Joules, and viscous dissipation on MHD flows and heat transfers of an incompressible fluid over a porous stretching sheet using a numerical method. They noted that temperature increase as the Eckert number increased. Kishan and Deepa [40] examined the effect of micropolar nanofluid flow and heat transfer on the porous flat plate with the viscous dissipation. Alim et al. [41] examined the impacts of viscous and Joule heating on the thermo-fluid dynamic field on a vertical flat surface. Ferdows et al. [42] evaluated the effects of viscous dissipation on the magnetohydrodynamics free convection flow of a compressible viscous fluids over a continuous sheet with Hall current. More specific details of the subject are available in the literature [43-49].

The main objective of this study is to investigate the stratified MHD boundary-layer flow of Jeffery nanofluid on a continuous surface with nanoparticles and gyrotactic micro-organisms. In fact, this kind of effort for non-Newtonian model should get even more specific. As a result, the primary focus in this study was put on assessing the fluid flow of Jeffery nanofluid on a continuous surface. The novelty of this study is given as follows:

i. Incorporation of gyrotactic micro-organisms;

ii. Consideration of the effects of magnetohydrodynamics; iii. Analysis of the flow with consideration of the viscous dissipation, Brownian motion, Joule heating, and thermophoresis; and

iv. The assumption of thermal, solutal, and motile density stratification aspects.

Flow analysis was taken out after converting the equations administering the flow into a system of Ordinary Differential Equations (ODEs). Then, a numerical solution was found using robust Keller box technique. The numerical results of the dimensionless velocity, temperature, density of nanoparticles, and concentration of motile micro-organism profiles for different essential parameters were graphically demonstrated. Finally, graphical and numerical results for skins friction, Nusselt number, Sherwood number, and motile microorganism number were also obtained and studied.

\section{Mathematical formulation}

A steady 2D laminar flow of an incompressible Jeffery nanofluid fluid was also investigated that resulted from a stretching surface coinciding with the planes $y=$ 0 . Furthermore, the effects of magnetohydrodynamics and nanoparticles were examined along with the gyrotactic microorganisms. Moreover, the effects of thermophoresis and Brownian motion were taken into consideration. The unvarying magnetic field $B_{0}$ was employed parallel to $y$-direction, and the fluid was confined to the region $y>0$ (see Figure 1). Here, $x$-axis is along the stretching sheet, and the flow is assumed to be produced by stretching the sheet with linear velocity of $U_{w}=c x$ away from the leading edge, where $c$ is a positive constant. The transfer rates of temperature, concentration, and motile microorganisms were analyzed under stratification effects. The induced magnetic field was neglected due to

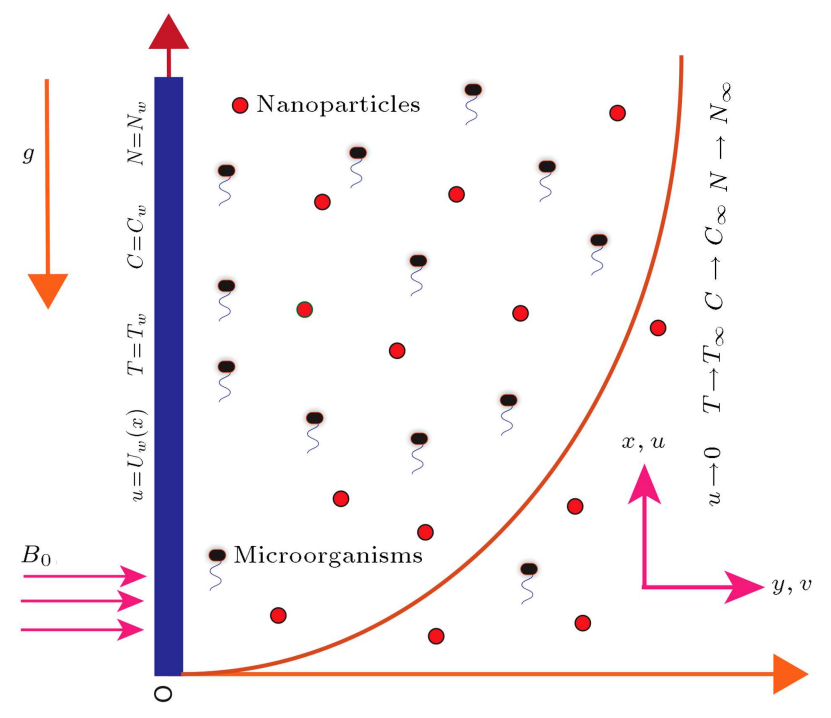

Figure 1. Physical model of the flow. 
its quite smaller value than the magnetic Reynolds number. Through boundary layer approximation, the continuity, momentum, energy, concentration, and microorganisms [50] equations are given in the following:

$$
\begin{aligned}
\left(u \frac{\partial u}{\partial x}+v \frac{\partial u}{\partial y}\right)=\frac{\nu}{\left(1+\lambda_{1}\right)}\left[\frac{\partial^{2} u}{\partial y^{2}}\right. & \\
+ & \left.\lambda_{2}\left(u \frac{\partial^{3} u}{\partial x \partial^{2} y}-\frac{\partial u}{\partial x} \frac{\partial^{2} u}{\partial y^{2}}+\frac{\partial u}{\partial y} \frac{\partial^{2} u}{\partial x \partial y}+v \frac{\partial^{3} u}{\partial y^{3}}\right)\right] \\
& -\frac{\sigma_{f}}{\rho_{f}} B_{0}^{2} u+\frac{g}{\rho_{f}}\left[\left(1-C_{\infty}\right) \rho_{f} \beta^{*}\left(T-T_{\infty}\right)\right. \\
- & \left.\left(\rho_{p}-\rho_{f}\right)\left(C-C_{\infty}\right)-\left(N-N_{\infty}\right) \gamma^{*}\left(\rho_{m}-\rho_{f}\right)\right]
\end{aligned}
$$$$
u \frac{\partial T}{\partial x}+v \frac{\partial T}{\partial y}=\alpha \frac{\partial^{2} T}{\partial y^{2}}
$$$$
+\tau\left(D_{B}\left(\frac{\partial C}{\partial y}\right)\left(\frac{\partial T}{\partial y}\right)+\frac{D_{T}}{T_{\infty}}\left(\frac{\partial T}{\partial y}\right)^{2}\right)
$$$$
+\frac{\sigma_{f}}{\left(\rho c_{p}\right)_{f}} B_{0}^{2} u^{2}+\frac{\mu}{\left(\rho c_{p}\right)_{f}\left(1+\lambda_{1}\right)}
$$$$
\left(\lambda_{2}\left(u \frac{\partial u}{\partial y} \frac{\partial^{2} u}{\partial x \partial y}+v \frac{\partial u}{\partial y} \frac{\partial^{2} u}{\partial y^{2}}\right)+\left(\frac{\partial u}{\partial y}\right)^{2}\right)
$$$$
u \frac{\partial C}{\partial x}+v \frac{\partial C}{\partial y}=D_{B} \frac{\partial^{2} C}{\partial y^{2}}+\frac{D_{T}}{T_{\infty}}\left(\frac{\partial^{2} T}{\partial y^{2}}\right),
$$$$
u \frac{\partial N}{\partial x}+v \frac{\partial N}{\partial y}+\frac{b W_{c}}{\left(C_{w}-C_{0}\right)} \frac{\partial}{\partial y}\left(N \frac{\partial C}{\partial y}\right)=D_{m} \frac{\partial^{2} N}{\partial y^{2}}
$$

The set of associated boundary conditions can be defined as:

$$
\begin{aligned}
& u=u_{w}(x)=c x, \quad v=0, \quad T=T_{w}=T_{\circ}+A_{1} x, \\
& C=C_{w}=C_{\circ}+E_{1} x, \quad N=N_{w}=N_{\circ}+H_{1} x \\
& u \longrightarrow 0, \quad T \longrightarrow T_{\infty}=T_{\circ}+A_{2} x, \\
& \text { at } y=0, \\
& C \longrightarrow C_{\infty}=C_{\circ}+E_{2} x, \quad N \longrightarrow N_{\infty}=N_{\circ}+H_{2} x \\
& \text { as } y \longrightarrow \infty,
\end{aligned}
$$

where $A_{1}, A_{2}, E_{1}, E_{2}, H_{1}$, and $H_{2}$ are the dimensional constants.

The following similarity transformation [50] was employed to convert Eqs. (1)-(5) to ODEs:

$$
\begin{aligned}
& \eta=\left(\frac{c}{\nu}\right)^{0.5} y, \quad \psi=-(c \nu)^{0.5} x f(\eta), \quad \theta=\frac{T-T_{\infty}}{T_{w}-T_{0}}, \\
& \phi=\frac{C-C_{\infty}}{C_{w}-C_{0}}, \quad \chi=\frac{N-N_{\infty}}{N_{w}-N_{0}},
\end{aligned}
$$

where $\eta, f, \theta, \phi, \chi$, and $\psi$ are similarity variable, dimensionless stream function, dimensionless temperature, dimensionless concentration of nanoparticles, dimensionless concentration of microorganisms and stream function obeying $u=\frac{\partial \psi}{\partial y}$ and $v=-\frac{\partial \psi}{\partial x}$, respectively. As a, result, we have:

$$
u=c x f^{\prime}(\eta), \quad v=-\sqrt{c \nu} f(\eta)
$$

where primes refers to the derivative with respect to $\eta$. Now, based on transformations from Eqs. (7) and (8), the subsequent set of non-linear ODEs can be obtained:

$$
\begin{aligned}
& f^{\prime \prime \prime}-\left(1+\lambda_{1}\right)\left[\left(f^{\prime}\right)^{2}-f f^{\prime \prime}\right]+\beta\left[\left(f^{\prime \prime}\right)^{2}-f f^{i v}\right] \\
& -\left(1+\lambda_{1}\right) M f^{\prime}+G r[\theta-N r \phi-R b \chi]=0,
\end{aligned}
$$

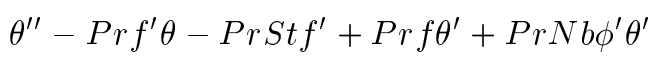

$$
\begin{aligned}
& +\operatorname{PrNt}\left(\theta^{\prime}\right)^{2}+\operatorname{Pr} E c\left(f^{\prime \prime}\right)^{2}+E c \operatorname{Pr} M\left(f^{\prime}\right)^{2}=0,
\end{aligned}
$$

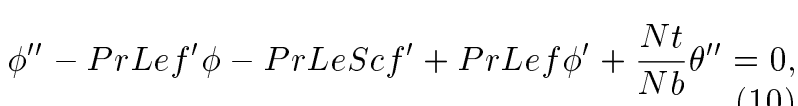

$$
\begin{aligned}
& \chi^{\prime \prime}-L b f^{\prime} \chi-L b S m f^{\prime}+L b f \chi^{\prime} \\
& -\operatorname{Pe}\left(\chi^{\prime} \phi^{\prime}+\phi^{\prime \prime}(\sigma+\chi)\right)=0 .
\end{aligned}
$$

According to similarity transform, the set of boundary conditions can be converted into the following form:

$$
\begin{aligned}
& f(0)=0, \quad f^{\prime}(0)=1, \quad \theta(0)=1-S t, \phi(0)=1-S c, \\
& \chi(0)=1-S m \text { at } \eta=0, \\
& f^{\prime}(\eta) \longrightarrow 0, \quad f^{\prime \prime}(\eta) \longrightarrow 0, \quad \theta(\eta) \longrightarrow 0, \phi(\eta) \longrightarrow 0, \\
& \chi(\eta) \longrightarrow 0 \text { as } \eta \longrightarrow \infty .
\end{aligned}
$$

Different dimensionless parameters appearing in Eqs. (9)-(14) are given below:

Bioconvection Peclet number:

$$
P e=\frac{b W_{c}}{D_{m}},
$$

Magnetic parameter:

$$
M=\frac{\sigma_{f} B_{0}^{2}}{a \rho}
$$

Eckert number:

$$
E c=\frac{c^{2} x^{2}}{c_{p}\left(T_{w}-T_{o}\right)},
$$

Bioconvection Lewis number:

$$
L b=\frac{\nu}{D_{m}}
$$

Thermophoresis parameter:

$$
N t=\frac{(\rho c)_{p} D_{T}\left(T_{w}-T_{o}\right)}{T_{\infty} \nu(\rho c)_{f}},
$$

Brownian motion parameter: 


$$
N b=\frac{(\rho c)_{p} D_{B}\left(C_{w}-C_{o}\right)}{\nu(\rho c)_{f}},
$$

Motile density stratification parameter:

$$
S m=\frac{H_{2}}{H_{1}},
$$

Deborah number:

$$
\beta=a \lambda_{2},
$$

Thermal stratification parameter:

$$
S t=\frac{A_{2}}{A_{1}},
$$

Lewis number:

$$
L e=\frac{\alpha}{D_{B}},
$$

Mass stratification parameter:

$$
S c=\frac{E_{2}}{E_{1}},
$$

Prandtl number:

$$
\operatorname{Pr}=\frac{\nu}{\alpha},
$$

Microorganisms concentration difference parameter:

$$
\sigma=\frac{N_{\infty}}{N_{w}-N_{0}},
$$

Mixed convection parameter:

$$
G r=\frac{\beta^{*} \gamma^{*}\left(1-C_{\infty}\right)\left(T_{w}-T_{o}\right)}{a U_{o}},
$$

Buoyancy ratio parameter:

$$
N r=\frac{\left(\rho_{p}-\rho_{f}\right)\left(C_{w}-C_{o}\right)}{\beta^{*} \rho_{f}\left(T_{w}-T_{o}\right)},
$$

Bioconvection Rayleigh number:

$$
R b=\frac{\gamma^{*}\left(N_{w}-N_{o}\right)\left(\rho_{m}-\rho_{f}\right)}{\beta^{*} \rho_{f}\left(1-C_{\infty}\right)\left(T_{w}-T_{o}\right)} .
$$

The valuable physicals quantities in this study include the local shear stress coefficient $C_{f}$, Nusselt number $N u_{x}$, Sherwood number $S h_{x}$, and density number $N n_{x}$ :

$$
\begin{aligned}
& C_{f}=\frac{2 \tau_{w}}{\rho_{f} U_{w}^{2}}, \quad N u_{x}=\frac{x q_{w}}{k\left(T_{w}-T_{0}\right)}, \\
& S h_{x}=\frac{x q_{m}}{D_{B}\left(C_{w}-C_{0}\right)}, \quad N n_{x}=\frac{x q_{n}}{D_{m}\left(N_{w}-N_{0}\right)},
\end{aligned}
$$

where:

$$
\begin{aligned}
& \tau_{w}=\left|\mu \frac{\partial u}{\partial y}\right|_{y=0}, \quad q_{w}=\left|-k \frac{\partial T}{\partial y}\right|_{y=0}, \\
& q_{m}=\left|-D_{B} \frac{\partial C}{\partial y}\right|_{y=0}, \quad q_{n}=\left|-D_{m} \frac{\partial N}{\partial y}\right|_{y=0} .
\end{aligned}
$$

Based on the aforementioned similarity transformation, Eq. (15) can be described as:

$$
\begin{aligned}
& \frac{1}{2} C_{f} R e_{x}^{0.5}=f^{\prime \prime}(0), \quad N u_{x} R e_{x}^{-0.5}=-\theta^{\prime}(0), \\
& S h_{x} R e_{x}^{-0.5}=-\phi^{\prime}(0), \quad N n_{x} R e_{x}^{-0.5}=-\chi^{\prime}(0),
\end{aligned}
$$

in which the local Reynolds numbers is $R e_{x}=\frac{x U_{w}}{\nu}$.

\section{Numerical solution by Keller box method}

To obtain the numerical solution of the nonlinear ODEs (Eqs. (9)-(12)) alongside the end point Conditions (Eq. (13)), Keller-box method [51,52] was employed and applied to different values for the involved parameters. The following steps are the main components of this numerical scheme:

(i) Decomposition of the momentum, energy, concentration, and microorganism equations into the first-order set of differential equations;

(ii) Achievement of difference equations through central finite differences;

(iii) Linearization of non-linear difference equations using Newton's idea;

(iv) Implementation of the block tri-diagonals elimination scheme to obtain the solution of the linear system iteratively. To this end, the new variables $\breve{u}_{1}, \breve{u}_{2}, \breve{u}_{3}, \breve{t}, \breve{c}$, and $\breve{g}$ were introduced to get a system of the first-order ODEs such that:

$$
\begin{aligned}
& \frac{d f}{d \eta}=\breve{u}_{1}, \quad \frac{d \breve{u}_{1}}{d \eta}=\breve{u}_{2}, \quad \frac{d \breve{u}_{2}}{d \eta}=\breve{u}_{3}, \\
& \frac{d \theta}{d \eta}=\breve{t}, \quad \frac{d \phi}{d \eta}=\breve{c}, \quad \frac{d \chi}{d \eta}=\breve{g} .
\end{aligned}
$$

The resulting system of the first-order ODEs is then written as:

$$
\begin{aligned}
-\beta f \frac{d \breve{u}_{3}}{d \eta} & +\breve{u}_{3}-\left(1+\lambda_{1}\right)\left[\breve{u}_{1}^{2}-f \breve{u}_{2}\right]+\beta \breve{u}_{2}^{2} \\
& -\left(1+\lambda_{1}\right) M \breve{u}_{1}+G r[\theta-N r \phi-R b \chi] \\
& =0,
\end{aligned}
$$

$$
\begin{aligned}
& \frac{d \breve{t}}{d \eta}-\operatorname{PrSt} \breve{u}_{1}-\operatorname{Pr} \breve{u}_{1} \theta+\operatorname{Prf} \breve{t} \\
& +\operatorname{PrNt}(\breve{t})^{2}+\operatorname{PrNb} \breve{c} \breve{t}+\operatorname{PrEc} \breve{u}_{2}^{2} \\
& +M \operatorname{PrEc} \breve{u}_{1}^{2}=0,
\end{aligned}
$$

$$
\begin{aligned}
& \frac{d \breve{c}}{d \eta}-\operatorname{Pr} \operatorname{Le} \breve{u}_{1} \phi-\operatorname{Pr} \operatorname{LeSc} \breve{u}_{1}+\operatorname{Pr} \operatorname{Lef} \breve{c}+\frac{N t}{N b} \frac{d \breve{t}}{d \eta}=0, \\
& \frac{d \breve{g}}{d \eta}-\operatorname{Lbu}_{1} \chi-\operatorname{LbSm}_{1}+\operatorname{Lbf\breve {g}} \\
& \quad-\operatorname{Pe}\left(g \breve{c}+(\sigma+\chi) \frac{d \breve{c}}{d \eta}\right)=0 .
\end{aligned}
$$




$$
\left.\begin{array}{l}
f(0)=0, \quad \breve{u}_{1}(0)=1, \quad \theta(0)=1-S t, \quad \phi(0)=1-S c, \quad \chi(0)=1-S m, \\
\breve{u}_{1} \rightarrow 0, \quad \breve{u}_{2} \rightarrow 0, \quad \theta \rightarrow 0, \quad \phi \rightarrow 0, \quad \chi \rightarrow 0 \quad \text { as } \quad \eta \rightarrow \infty .
\end{array}\right\}
$$

Box I

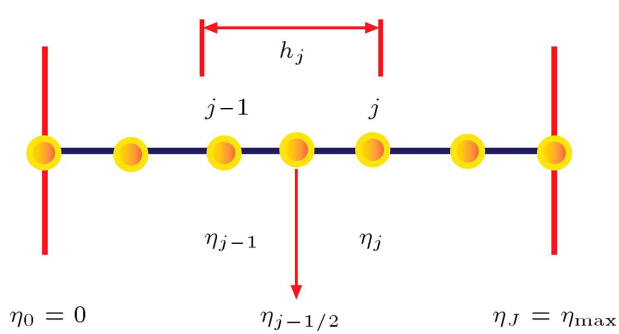

Figure 2. Difference approximation to the grid structure for the Keller box scheme.

The evolved boundary conditions for this study are given by Eq. (22) as shown in Box I. A one dimensional computational grid was also employed as shown in Figure 2. The domain was discretized with the help of the subsequent nodes:

$$
\eta_{0}=0, \quad \eta_{j}=\eta_{j-1}+h_{j}, j=0,1,2,3 \ldots, J, \quad \eta_{J}=\eta_{\infty},
$$

where $h_{j}$ is the step size. The central difference approximations, as pointed out in (ii), are as follows:

$$
\begin{aligned}
& \frac{f_{j}-f_{j-1}}{h_{j}}=\frac{\left(\breve{u}_{1}\right)_{j}+\left(\breve{u}_{1}\right)_{j-1}}{2}, \\
& \frac{\left(\breve{u}_{1}\right)_{j}-\left(\breve{u}_{1}\right)_{j-1}}{2}=\frac{\left(\breve{u}_{2}\right)_{j}+\left(\breve{u}_{2}\right)_{j-1}}{2}, \\
& \frac{\left(\breve{u}_{2}\right)_{j}-\left(\breve{u}_{2}\right)_{j-1}}{2}=\frac{\left(\breve{u}_{3}\right)_{j}+\left(\breve{u}_{3}\right)_{j-1}}{2}, \\
& \frac{\theta_{j}-\theta_{j-1}}{h_{j}}=\frac{\breve{t}_{j}+\underline{r} e v e t_{j-1}}{2}, \\
& \frac{\phi_{j}-\phi_{j-1}}{h_{j}}=\frac{\breve{c}_{j}+\breve{c}_{j-1}}{2}, \\
& \frac{\chi_{j}-\chi_{j-1}}{h_{j}}=\frac{\breve{g}_{j}+\breve{g}_{j-1}}{2},
\end{aligned}
$$

Eqs. (29)-(32) are shown in Box II. To linearize the system of nonlinear equations (Eqs. (24)-(33)) through Newton's method, the following substitutions are suggested:

$$
\begin{aligned}
\left(\breve{u}_{1}\right)_{j}^{n+1} & =\left(\breve{u}_{1}\right)_{j}^{n}+\delta\left(\breve{u}_{1}\right)_{j}^{n}, \quad\left(\breve{u}_{2}\right)_{j}^{n+1}=\left(\breve{u}_{2}\right)_{j}^{n}+\delta\left(\breve{u}_{2}\right)_{j}^{n}, \\
\left(\breve{u}_{3}\right)_{j}^{n+1} & =\left(\breve{u}_{3}\right)_{j}^{n}+\delta\left(\breve{u}_{3}\right)_{j}^{n},
\end{aligned}
$$

$$
\begin{array}{ll}
f_{j}^{n+1}=f_{j}^{n}+\delta f_{j}^{n}, & \theta_{j}^{n+1}=\theta_{j}^{n}+\delta \theta_{j}^{n}, \\
\phi_{j}^{n+1}=\phi_{j}^{n}+\delta \phi_{j}^{n}, & \chi_{j}^{n+1}=\chi_{j}^{n}+\delta \chi_{j}^{n}, \\
t_{j}^{n+1}=t_{j}^{n}+\delta t_{j}^{n}, & c_{j}^{n+1}=c_{j}^{n}+\delta c_{j}^{n}, \\
g_{j}^{n+1}=g_{j}^{n}+\delta g_{j}^{n} . &
\end{array}
$$

By substituting these expressions into Eqs. (23)-(32) and dropping the higher-order terms in $\delta$, the following system is obtained as:

$$
\begin{aligned}
& \left(\delta f_{j}-\delta f_{j-1}\right)-\frac{h_{j}}{2}\left(\delta\left(\breve{u}_{1}\right)_{j}+\delta\left(\breve{u}_{1}\right)_{j-1}\right)=\left(r_{1}\right)_{j}, \\
& \left(\delta\left(\breve{u}_{1}\right)_{j}-\delta\left(\breve{u}_{1}\right)_{j-1}\right)-\frac{h_{j}}{2}\left(\delta\left(\breve{u}_{2}\right)_{j}+\delta\left(\breve{u}_{2}\right)_{j-1}\right)=\left(r_{2}\right)_{j}
\end{aligned}
$$

$$
\left(\delta\left(\breve{u}_{2}\right)_{j}-\delta\left(\breve{u}_{2}\right)_{j-1}\right)-\frac{h_{j}}{2}\left(\delta\left(\breve{u}_{3}\right)_{j}+\delta\left(\breve{u}_{3}\right)_{j-1}\right)=\left(r_{3}\right)_{j},
$$

$$
\begin{gathered}
\left(\delta \theta_{j}-\delta \theta_{j-1}\right)-\frac{h_{j}}{2}\left(\delta \breve{t}_{j}+\delta \breve{t}_{j-1}\right)=\left(r_{4}\right)_{j}, \\
\left(\delta \phi_{j}-\delta \phi_{j-1}\right)-\frac{h_{j}}{2}\left(\delta \breve{c}_{j}+\delta \breve{c}_{j-1}\right)=\left(r_{5}\right)_{j}, \\
\left(\delta \chi_{j}-\delta \chi_{j-1}\right)-\frac{h_{j}}{2}\left(\delta \breve{g}_{j}+\delta \breve{g}_{j-1}\right)=\left(r_{6}\right)_{j}, \\
\left(\xi_{1}\right)_{j} \delta\left(\breve{u}_{3}\right)_{j}+\left(\xi_{2}\right)_{j} \delta\left(\breve{u}_{3}\right)_{j-1}+\left(\xi_{3}\right)_{j} \delta f_{j}+\left(\xi_{4}\right)_{j} \delta f_{j-1} \\
+\left(\xi_{5}\right)_{j} \delta\left(\breve{u}_{2}\right)_{j}+\left(\xi_{6}\right)_{j} \delta\left(\breve{u}_{2}\right)_{j-1}+\left(\xi_{7}\right)_{j} \delta\left(\breve{u}_{1}\right)_{j} \\
+\left(\xi_{8}\right)_{j} \delta\left(\breve{u}_{1}\right)_{j-1}+\left(\xi_{9}\right)_{j} \delta \theta_{j}+\left(\xi_{10}\right)_{j} \delta \theta_{j-1} \\
+\left(\xi_{11}\right)_{j} \delta \phi_{j}+\left(\xi_{12}\right)_{j} \delta \phi_{j-1}+\left(\xi_{13}\right)_{j} \delta \chi_{j} \\
+\left(\xi_{14}\right)_{j} \delta \chi_{j-1}=\left(r_{7}\right)_{j},
\end{gathered}
$$

$$
\begin{aligned}
\left(\beta_{1}\right)_{j} \delta \breve{t}_{j} & +\left(\beta_{2}\right)_{j} \delta \breve{t}_{j-1}+\left(\beta_{3}\right)_{j} \delta \breve{c}_{j}+\left(\beta_{4}\right)_{j} \delta \breve{c}_{j-1} \\
& +\left(\beta_{5}\right)_{j} \delta f_{j}+\left(\beta_{6}\right)_{j} \delta f_{j-1}+\left(\beta_{7}\right)_{j} \delta \theta_{j} \\
& +\left(\beta_{8}\right)_{j} \delta \theta_{j-1}+\left(\beta_{9}\right)_{j} \delta\left(\breve{u}_{1}\right)_{j}+\left(\beta_{10}\right)_{j} \delta\left(\breve{u}_{1}\right)_{j-1} \\
& +\left(\beta_{11}\right)_{j} \delta\left(\breve{u}_{2}\right)_{j}+\left(\beta_{12}\right)_{j} \delta\left(\breve{u}_{2}\right)_{j-1}=\left(r_{8}\right)_{j},
\end{aligned}
$$




$$
\begin{aligned}
& \beta\left[\left(\frac{\left(\breve{u}_{2}\right)_{j}+\left(\breve{u}_{2}\right)_{j-1}}{2}\right)^{2}-\left(\frac{f_{j}+f_{j-1}}{2}\right)\left(\frac{\left(\breve{u}_{3}\right)_{j}-\left(\breve{u}_{3}\right)_{j-1}}{h_{j}}\right)\right]-\left(1+\lambda_{1}\right) \\
& \left.\left[\left(\frac{\left(\breve{u}_{1}\right)_{j}+\left(\breve{u}_{1}\right)_{j-1}}{2}\right)^{2}-\left(\frac{f_{j}+f_{j-1}}{2}\right)\left(\frac{\left(\breve{u}_{2}\right)_{j}+\left(\breve{u}_{2}\right)_{j-1}}{2}\right)\right]+\left(\frac{\left(\breve{u}_{3}\right)_{j}+\left(\breve{u}_{3}\right)_{j-1}}{2}\right)\right\} \\
& -M\left(1+\lambda_{1}\right)\left(\frac{\left(\breve{u}_{1}\right)_{j}+\left(\breve{u}_{1}\right)_{j-1}}{2}\right)+G r\left[\left(\theta_{j-\frac{1}{2}}\right)-N r\left(\phi_{j-\frac{1}{2}}\right)-R b\left(\chi_{j-\frac{1}{2}}\right)\right]=0, \quad \\
& \left(\frac{\breve{t}-\breve{t}_{j-1}}{h_{j}}\right)-\operatorname{Pr}\left(\frac{\left(\breve{u}_{1}\right)_{j}+\left(\breve{u}_{1}\right)_{j-1}}{2}\right)\left(\frac{\theta_{j}+\theta_{j-1}}{2}\right)-\operatorname{PrSt}\left(\frac{\left(\breve{u}_{1}\right)_{j}+\left(\breve{u}_{1}\right)_{j-1}}{2}\right)+ \\
& \left.\operatorname{Pr}\left(\frac{f_{j}+f_{j-1}}{2}\right)\left(\frac{\breve{t}_{j}+\breve{t}_{j-1}}{2}\right)+\operatorname{PrNb}\left(\frac{\breve{c}_{j}+\breve{c}_{j-1}}{2}\right)\left(\frac{\breve{t}_{j}+\breve{t}_{j-1}}{2}\right)+\operatorname{PrNt}\left(\frac{\breve{t}_{j}+\breve{t}_{j-1}}{2}\right)^{2}\right\} \\
& +\operatorname{PrEc}\left(\frac{\left(\breve{u}_{2}\right)_{j}+\left(\breve{u}_{2}\right)_{j-1}}{2}\right)^{2}+\operatorname{MPrEc}\left(\frac{\left(\breve{u}_{1}\right)_{j}+\left(\breve{u}_{1}\right)_{j-1}}{2}\right)^{2}=0 \\
& \left.\left(\frac{\breve{c}-\breve{c}_{j-1}}{h_{j}}\right)-\operatorname{Pr} \operatorname{Le}\left(\frac{\left(\breve{u}_{1}\right)_{j}+\left(\breve{u}_{1}\right)_{j-1}}{2}\right)\left(\frac{\phi_{j}+\phi_{j-1}}{2}\right)-\operatorname{Pr} \operatorname{LeSc}\left(\frac{\left(\breve{u}_{1}\right)_{j}+\left(\breve{u}_{1}\right)_{j-1}}{2}\right)\right) \\
& +\operatorname{PrLe}\left(\frac{f_{j}+f_{j-1}}{2}\right)\left(\frac{\breve{c}_{j}+\breve{c}_{j-1}}{2}\right)+\frac{N t}{N b}\left(\frac{\breve{t}-\breve{t}_{j-1}}{h_{j}}\right)=0 \\
& \left.\left(\frac{\breve{g}-\breve{g}_{j-1}}{h_{j}}\right)-L b\left(\frac{\left(\breve{u}_{1}\right)_{j}+\left(\breve{u}_{1}\right)_{j-1}}{2}\right)\left(\frac{\chi_{j}+\chi_{j-1}}{2}\right)-\operatorname{LbSm}\left(\frac{\left(\breve{u}_{1}\right)_{j}+\left(\breve{u}_{1}\right)_{j-1}}{2}\right)+\right) \\
& L b\left(\frac{f_{j}+f_{j-1}}{2}\right)\left(\frac{\breve{g}_{j 1}+\breve{g}_{j-1}}{2}\right)-\operatorname{Pe}\left(\frac{\breve{g}_{j}+\breve{g}_{j-1}}{2}\right)\left(\frac{\breve{c}_{j}+\breve{c}_{j-1}}{2}\right) \\
& -P e\left(\sigma+\frac{f_{j}+f_{j-1}}{2}\right)\left(\frac{\breve{c}-\breve{c}_{j-1}}{h_{j}}\right)=0
\end{aligned}
$$

Box II

$$
\begin{aligned}
\left(\alpha_{1}\right)_{j} & \delta \breve{c}_{j}+\left(\alpha_{2}\right)_{j} \delta \breve{c}_{j-1}+\left(\alpha_{3}\right)_{j} \delta f_{j}+\left(\alpha_{4}\right)_{j} \delta f_{j-1} \\
& +\left(\alpha_{5}\right)_{j} \delta\left(\breve{u}_{1}\right)_{j}+\left(\alpha_{6}\right)_{j} \delta\left(\breve{u}_{1}\right)_{j-1}+\left(\alpha_{7}\right)_{j} \delta \phi_{j} \\
& +\left(\alpha_{8}\right)_{j} \delta \phi_{j-1}+\left(\alpha_{9}\right)_{j} \delta t_{j}+\left(\alpha_{10}\right)_{j} \delta t_{j-1} \\
& =\left(r_{9}\right)_{j}
\end{aligned}
$$

$\left(\gamma_{1}\right)_{j} \delta \breve{c}_{j}+\left(\gamma_{2}\right)_{j} \delta \breve{c}_{j-1}+\left(\gamma_{3}\right)_{j} \delta \chi_{j}+\left(\gamma_{4}\right)_{j} \delta \chi_{j-1}$

$$
\begin{aligned}
& +\left(\gamma_{5}\right)_{j} \delta f_{j}+\left(\gamma_{6}\right)_{j} \delta f_{j-1}+\left(\gamma_{7}\right)_{j} \delta \breve{g}_{j} \\
& +\left(\gamma_{8}\right)_{j} \delta \breve{g}_{j-1}=\left(r_{10}\right)_{j},
\end{aligned}
$$

where:

$$
\left(\xi_{1}\right)_{j}=\frac{h_{j}}{2}-\frac{\beta}{2}\left(f_{j}+f_{j-1}\right), \quad\left(\xi_{2}\right)_{j}=\frac{h_{j}}{2}+\frac{\beta}{2}\left(f_{j}+f_{j-1}\right),
$$

$$
\begin{aligned}
\left(\xi_{3}\right)_{j}= & \frac{h_{j}\left(1+\lambda_{1}\right)}{4}\left(\left(\breve{u}_{2}\right)_{j}+\left(\breve{u}_{2}\right)_{j-1}\right)-\frac{\beta}{2}\left(\left(\breve{u}_{3}\right)_{j}\right. \\
& \left.-\left(\breve{u}_{3}\right)_{j-1}\right)=\left(\xi_{4}\right)_{j}, \\
\left(\xi_{5}\right)_{j}= & \frac{h_{j}\left(1+\lambda_{1}\right)}{4}\left(f_{j}+f_{j-1}\right)+\frac{h_{j} \beta}{2}\left(\left(\breve{u}_{2}\right)_{j}+\right. \\
& \left.\left(\breve{u}_{2}\right)_{j-1}\right)=\left(\xi_{6}\right)_{j}, \\
\left(\xi_{7}\right)_{j}= & \frac{-1}{2} h_{j} M\left(1+\lambda_{1}\right)-\frac{h_{j}\left(1+\lambda_{1}\right)}{2} b i g\left(\left(\breve{u}_{1}\right)_{j}\right. \\
& \left.+\left(\breve{u}_{1}\right)_{j-1}\right)=\left(\xi_{8}\right)_{j}, \\
\left(\xi_{9}\right)_{j}= & \frac{h_{j}}{2} G r=\left(\xi_{10}\right)_{j}, \quad\left(\xi_{11}\right)_{j}=-\frac{h_{j}}{2} G r N r=\left(\xi_{12}\right)_{j}, \\
\left(\xi_{13}\right)_{j}= & -\frac{h_{j}}{2} G r R b=\left(\xi_{14}\right)_{j},
\end{aligned}
$$




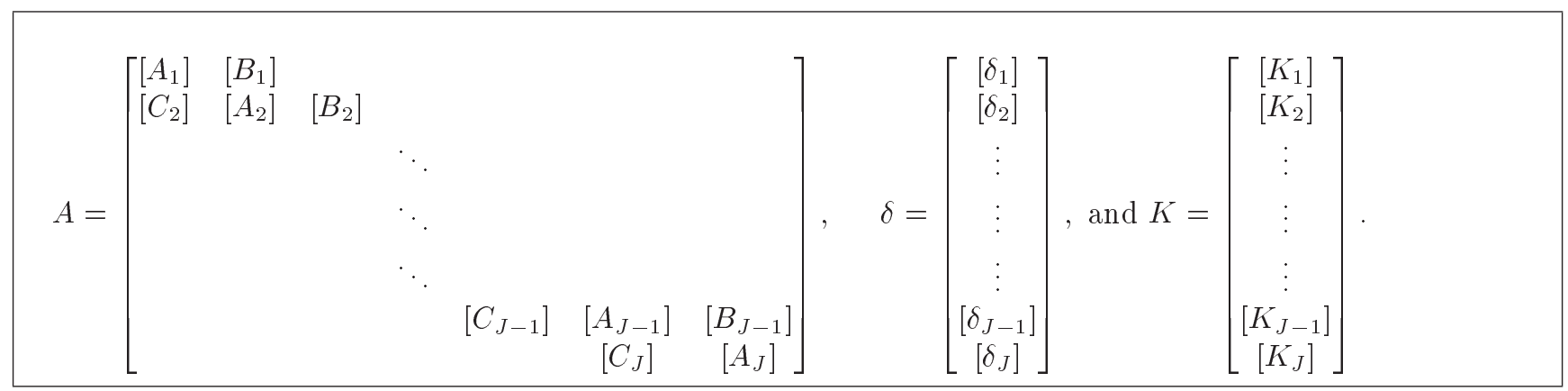

Box III

$$
\begin{aligned}
\left(r_{7}\right)_{j}= & \frac{h_{j}}{2} M\left(1+\lambda_{1}\right)\left(\breve{u}_{1}\right)_{j-\frac{1}{2}}+\frac{h_{j}}{4}\left(1+\lambda_{1}\right) \\
& \left(\breve{u}_{1}\right)_{j-\frac{1}{2}}^{2}-\frac{h_{j}}{4}\left(1+\lambda_{1}\right) f_{j-\frac{1}{2}}\left(\breve{u}_{2}\right)_{j-\frac{1}{2}} \\
& -\frac{1}{4} h_{j}\left(\breve{u}_{2}\right)_{j-\frac{1}{2}}^{2}-\frac{1}{2}\left(\breve{u}_{3}\right)_{j-\frac{1}{2}}+\frac{1}{2} \beta f_{j-\frac{1}{2}} \\
& \left(\left(\breve{u}_{3}\right)_{j}-\left(\breve{u}_{3}\right)_{j-1}\right)-G r h_{j} \theta_{j-\frac{1}{2}}+G r N r h_{j} \phi_{j-\frac{1}{2}} \\
& +G r R b h_{j} \chi_{j-\frac{1}{2}} . \\
\left(\beta_{1}\right)_{j}= & 1+\frac{h_{j} N_{b} P r}{4}\left(\breve{c}+\breve{c}_{j-1}\right)+\frac{h_{j} P r}{4}\left(f_{j}+f_{j-1}\right) \\
& +\frac{h_{j} N_{t} P r}{2}\left(\breve{t}+\breve{t}_{j-1}\right),
\end{aligned}
$$

$\left(\beta_{2}\right)_{j}=\left(\beta_{1}\right)_{j}-2, \quad\left(\beta_{3}\right)_{j}=\frac{h_{j} N_{b} P r}{4}\left(\breve{t}+\breve{t}_{j-1}\right)=\left(\beta_{4}\right)_{j}$,

$\left(\beta_{5}\right)_{j}=\frac{h_{j} P r}{4}\left(\breve{t}_{j}+\breve{t}_{j-1}\right)=\left(\beta_{6}\right)_{j}$,

$\left(\beta_{7}\right)_{j}=-\operatorname{Prh}_{j} \frac{\left(\left(\breve{u}_{2}\right)_{j}+\left(\breve{u}_{2}\right)_{j-1}\right)}{2}=\left(\beta_{8}\right)_{j}$,

$\left(\beta_{9}\right)_{j}=\frac{h_{j} M \operatorname{PrEc}}{2}\left(\left(\breve{u}_{1}\right)_{j}+\left(\breve{u}_{1}\right)_{j-1}\right)-\frac{\operatorname{Pr} h_{j}}{2}\left(\theta_{j}\right.$

$$
\left.+\theta_{j-1}\right)=\left(\beta_{10}\right)_{j},
$$

$$
\left(r_{8}\right)_{j}=\left(\breve{t}_{j-1}-\breve{t}_{j}\right)-h_{j} N_{b} \operatorname{Pr}(\breve{c})_{j-\frac{1}{2}}(\breve{t})_{j-\frac{1}{2}}
$$$$
-h_{j} \operatorname{Pr}(\breve{t})_{j-\frac{1}{2}} f_{j-\frac{1}{2}}-h_{j} N_{t} \operatorname{Pr}(\breve{t})_{j-\frac{1}{2}}^{2}
$$$$
-h_{j} \operatorname{MPrEc}\left(\breve{u}_{1}\right)_{j-\frac{1}{2}}^{2}-h_{j} \operatorname{PrEc}\left(\breve{u}_{2}\right)_{j-\frac{1}{2}}^{2}
$$$$
-2 h_{j} \operatorname{Pr}\left(\breve{u}_{1}\right)_{j-\frac{1}{2}} \theta_{j-\frac{1}{2}} .
$$

$$
\left(\alpha_{1}\right)_{j}=1+\frac{h_{j} S c}{4}\left(f_{j}+f_{j-1}\right), \quad\left(\alpha_{2}\right)_{j}=\left(\alpha_{1}\right)_{j}-2,
$$$$
\left(\alpha_{3}\right)_{j}=\frac{h_{j} S c}{4}\left(\breve{c}+\breve{c}_{j-1}\right)=\left(\alpha_{4}\right)_{j},
$$

$$
\begin{aligned}
\left(\alpha_{7}\right)_{j}= & -\frac{h_{j} S c}{2}\left(\left(\breve{u}_{1}\right)_{j}+\left(\breve{u}_{1}\right)_{j-1}\right)=\left(\alpha_{8}\right)_{j}, \\
\left(\alpha_{9}\right)_{j}= & -S r=-\left(\alpha_{10}\right)_{j}, \\
\left(r_{9}\right)_{j}= & \left(\breve{c}_{j-1}-\breve{c}_{j}\right)+S r\left(\breve{t}_{j-1}-\breve{t}_{j}\right)-\frac{h_{j} S c}{4} \breve{c}_{j-\frac{1}{2}} f_{j-\frac{1}{2}} \\
& +\frac{h_{j} S c}{2}\left(\breve{u}_{1}\right)_{j-\frac{1}{2}} \phi_{j-\frac{1}{2}} .
\end{aligned}
$$

$$
\left(\gamma_{1}\right)_{j}=-p e \sigma-\frac{p e}{2}\left(\chi_{j}+\chi_{j-1}\right)-\frac{h_{j} p e}{4}\left(\breve{g}_{j}+\breve{g}_{j-1}\right),
$$$$
\left(\gamma_{2}\right)_{j}=p e \sigma-\frac{p e}{2}\left(\chi_{j}+\chi_{j-1}\right)-\frac{h_{j} p e}{4}\left(\breve{g}_{j}+\breve{g}_{j-1}\right),
$$$$
\left(\gamma_{3}\right)_{j}=\frac{p e}{2}\left(\breve{c}_{j}+\breve{c}_{j-1}\right)=\left(\gamma_{4}\right)_{j},
$$$$
\left(\gamma_{5}\right)_{j}=\frac{h_{j} L b}{4}\left(\breve{g}_{j}+\breve{g}_{j-1}\right)=\left(\gamma_{6}\right)_{j},
$$$$
\left(\gamma_{7}\right)_{j}=1-\frac{h_{j} p e}{4}\left(\breve{c}_{j}+\breve{c}_{j-1}\right)+\frac{h_{j} p e}{4}\left(f_{j}+\chi_{j-1}\right),
$$$$
\left(\gamma_{8}\right)_{j}=\left(\gamma_{7}\right)_{j}-2,
$$$$
\left(r_{10}\right)_{j}=\left(\breve{g}_{j-1}-\breve{g}_{j}\right)+\operatorname{pe\sigma }\left(\breve{c}_{j}-\breve{c}_{j-1}\right)
$$

$$
\begin{aligned}
& +p e\left(\breve{c}_{j}-\breve{c}_{j-1}\right) \chi_{j-\frac{1}{2}}+h_{j} p e \breve{c}_{j-\frac{1}{2}} \breve{g}_{j-\frac{1}{2}} \\
& -h_{j} L b \breve{g}_{j-\frac{1}{2}} f_{j-\frac{1}{2}} .
\end{aligned}
$$

After linearization, the following block tridiagonal system is obtained as:

$$
A \delta=K .
$$

$A, \delta$, and $K$ are shown in Box III and the entries of the matrices are provided in Box IV. Now we factorize $A$ as:

$$
A=L U,
$$

$\left(\alpha_{5}\right)_{j}=\frac{h_{j} S c}{2}\left(\phi_{j}+\phi_{j-1}\right)=-\left(\gamma_{6}\right)_{j}$,

where: 


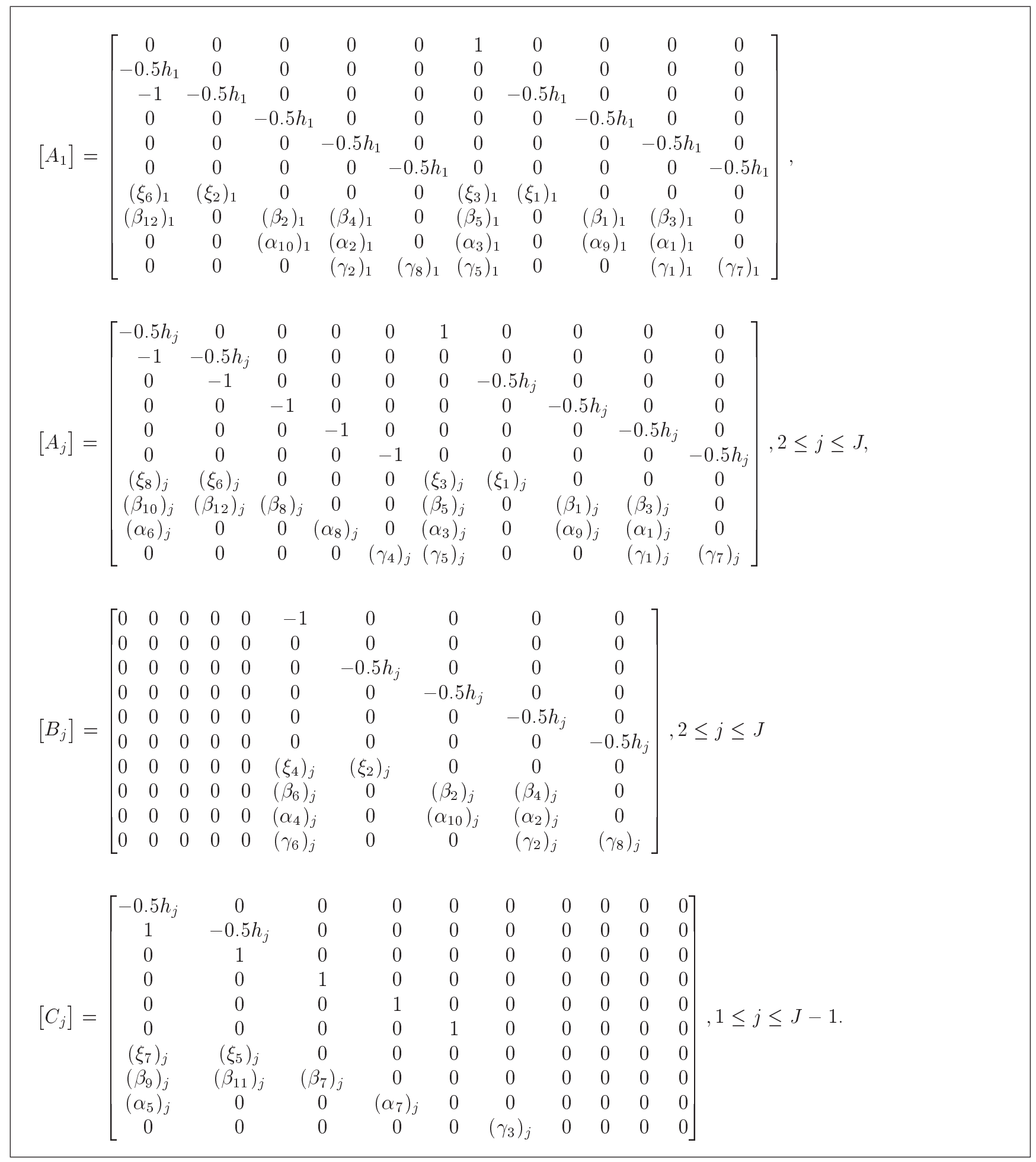

Box IV

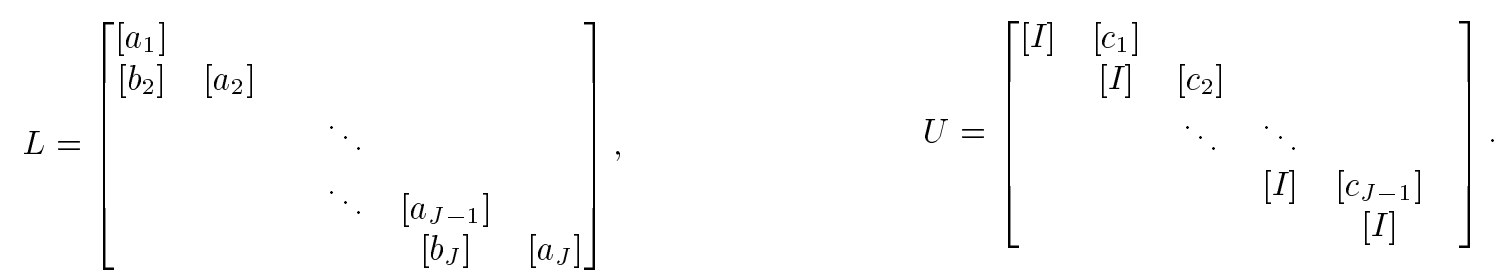


Table 1. Comparison of $\theta^{\prime}(0)$ for the case $G r=N r=R b=\beta=E c=M=L b=P e=N t=L e=0$.

\begin{tabular}{cccccc}
\hline $\boldsymbol{P r}$ & Present study & Ishak et al. [53] & Accuracy (\%) [53] & Pal and Mondal [54] & Accuracy (\%) [54] \\
\hline 1.0 & 1.337050 & 1.3333 & $99.71 \%$ & 1.333333 & $99.72 \%$ \\
3.0 & 2.500405 & 2.5097 & $99.62 \%$ & 2.509715 & $99.62 \%$ \\
10 & 4.798532 & 4.7969 & $99.96 \%$ & 4.796871 & $99.97 \%$ \\
\hline
\end{tabular}

Here, $\left[a_{i}\right],\left[b_{i}\right]$, and $\left[c_{i}\right]$ are $10 \times 10$ matrices and $[I]$ is the unit matrix of order 10. Eq. (48) provides the solution for $\delta$ by the $L U$ factorization method. The grid of size $h_{j}=0.001$ was employed to achieve the numerical solution using $\eta_{\max }=12$ and an error tolerance of $10^{-6}$ for all instances. To ensure the accuracy of the adopted numerical strategy, a highly convincing comparison of the present results with those reported by Ishak et al. [53] and Pal and Mondal [54] for $\theta^{\prime}(0)$ was conducted, the results of which are displayed in Table 1. The obtained numerical results in this study indicated upto $99.96 \%$ accuracy with those obtained by Ishak et al. [53] and upto $99.97 \%$ accuracy with those obtained from the Pal and Mondal [54].

\section{Results and discussions}

The evolved equations of velocity, temperature, concentration, and density of motile microorganisms (Eqs. (8)-(11)) together with the boundary condition (Eq. (12)) were numerically dealt with, and the computational analysis was carried out for some appropriate choices of the physical parameters. Table 2 presents the numerical values of local heat transfer, Sherwood number, and density of motile microorganisms for different values for the relevant parameters. According to this table, the enhancement of the magnetic number $M$, Brownian motion parameter $\mathrm{Nb}$, thermophoresis parameter $N t$, Eckert number $E c$, relaxation to the retardation time $\lambda$, and Lewis number $L e$ would reduce the Nusselt number on the surface; however, it could increase in the case of the escalating values of the Deborah number $\beta$ and Prandtl number $\operatorname{Pr}$. An intensification in the Brownian motion $\mathrm{Nb}$, thermophoresis $N t$, Deborah number $P r$, and Prandtl number $\operatorname{Pr}$ would diminish the Sherwood number, while it would increase in the case of the increasing values of the magnetic number $M$, Lewis number Le, relaxation to the retardation time $\lambda$ and Eckert number $E c$. In addition, a rise in the magnetic number $M$, Lewis number Le, Eckert number Ec, Peclet number $P e$, bioconvection Lewis number $L b$, relaxation to the retardation times $\lambda$, and concentrations difference parameter of micro-organisms $\sigma$ would increase the density rate of motile micro-organisms, yet it was reduced in the case of increase in the values of the Brownian motion $N b$, thermophoresis $N t$, Deborah number $\beta$, relaxation to the retardation time $\beta$, and
Prandtl number $P r$. Table 3 provides information on the sundry parameters $M$ on the Nusselt number in the case of conventional base fluid and nanofluid, respectively. According to this table, the heat transfer rate of $M$ is minimum $2.41 \%$ and maximum $15.68 \%$. Obviously, the aforementioned parameter is valuable in terms of heat transport in the case of nanofluid on a stretchable surface.

Figures 3-19 discuss the dynamics in the velocity profile $f^{\prime}(\eta)$, temperature $\theta(\eta)$, concentrations of nanoparticles $\phi(\eta)$, and density of motile microorganisms $\chi(\eta)$ of the nanofluid with different values of some important physical parameters such as Deborah number $\beta$, magnetic number $M$, Prandtl number $\operatorname{Pr}$, Eckert number $E c$, thermophoresis $N t$, Brownian motion $N b$, Peclet number $P e$, bioconvection Lewis numbers Lb, thermal stratification $S t$, motile density stratification $S m$, Lewis number Le, concentrations difference of micro-organisms $\sigma$, and concentrations stratification $S c$. In addition, Figures 20-25 examine the essential and relevant quantities of the physical interest, especially the local drag coefficient, Nusselt number, Sherwood number, and density rate of motile microorganisms.

Figure 3 examines the effect of $M$ on the velocity distribution $f^{\prime}(\eta)$. As observed, the presence of $M$ reduced the boundary-layer thickness as well as the velocity profile. Physically, the magnetic field creates a Lorentz force that gives an opposition to the fluid

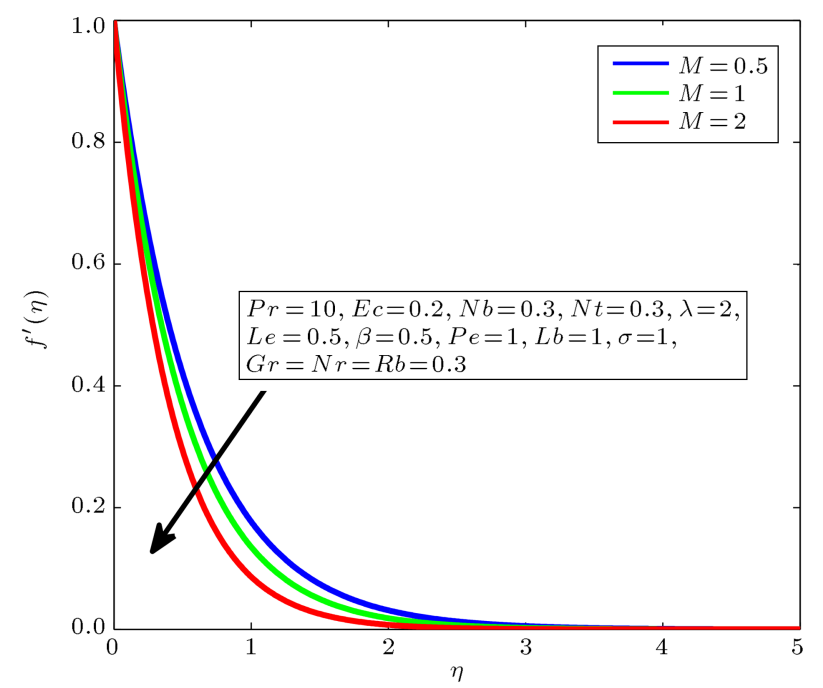

Figure 3. Influence of $M$ on $f^{\prime}(\eta)$. 
Table 2. Variation of $-\theta^{\prime}(0),-\phi^{\prime}(0)$ and $-\chi^{\prime}(0)$ for different types of parameters in flow.

\begin{tabular}{|c|c|c|c|c|c|c|c|c|c|c|c|c|c|}
\hline$M$ & $L e$ & $N t$ & $N b$ & $\boldsymbol{\beta}$ & $E c$ & $\boldsymbol{P r}$ & $P e$ & $L b$ & $\sigma$ & $\lambda$ & $-\theta^{\prime}(0)$ & $-\phi^{\prime}(0)$ & $-\chi^{\prime}(0)$ \\
\hline 0.5 & 1 & 0.1 & 0.1 & 0.5 & 0.5 & 10 & 1 & 1 & 1 & 2 & 1.48542 & 0.00685 & 0.46113 \\
\hline 1 & & & & & & & & & & & 0.74877 & 0.59253 & 1.55852 \\
\hline 1.5 & & & & & & & & & & & 0.05718 & 1.16240 & 2.64117 \\
\hline & 2 & & & & & & & & & & 0.76777 & 1.34107 & 2.96743 \\
\hline & 4 & & & & & & & & & & 0.64896 & 2.44760 & 5.07854 \\
\hline & 6 & & & & & & & & & & 0.58940 & 3.22067 & 6.57480 \\
\hline & & 0.1 & & & & & & & & & 0.89215 & 0.47672 & 1.34053 \\
\hline & & 0.2 & & & & & & & & & 0.79296 & 0.15883 & 0.86705 \\
\hline & & 0.3 & & & & & & & & & 0.72259 & 0.00386 & 0.72025 \\
\hline & & & 0.1 & & & & & & & & 0.89215 & 0.47672 & 1.34053 \\
\hline & & & 0.2 & & & & & & & & 0.79296 & 0.15883 & 0.86705 \\
\hline & & & 0.3 & & & & & & & & 0.72259 & 0.00386 & 0.72025 \\
\hline & & & & 0.1 & & & & & & & 0.29784 & 0.92415 & 2.17335 \\
\hline & & & & 0.5 & & & & & & & 0.89215 & 0.47672 & 1.34053 \\
\hline & & & & 1 & & & & & & & 1.32571 & 0.17185 & 0.77225 \\
\hline & & & & & 0.4 & & & & & & 1.18143 & 0.20785 & 0.81387 \\
\hline & & & & & 0.5 & & & & & & 0.60226 & 0.74615 & 1.86842 \\
\hline & & & & & 0.6 & & & & & & 0.02007 & 1.28732 & 2.93496 \\
\hline & & & & & & 1 & & & & & 0.49346 & 0.69420 & 1.66729 \\
\hline & & & & & & 2 & & & & & 0.65966 & 0.57279 & 1.41428 \\
\hline & & & & & & 3 & & & & & 0.77451 & 0.49256 & 1.22175 \\
\hline & & & & & & & 1 & & & & 0.89215 & 0.47672 & 1.34053 \\
\hline & & & & & & & 2 & & & & 0.89260 & 0.47648 & 2.23952 \\
\hline & & & & & & & 3 & & & & 0.89269 & 0.47649 & 3.15047 \\
\hline & & & & & & & & 0.5 & & & 0.89215 & 0.47672 & 1.15028 \\
\hline & & & & & & & & 1 & & & 0.89215 & 0.47672 & 1.34053 \\
\hline & & & & & & & & 1.5 & & & 0.89215 & 0.47672 & 1.50766 \\
\hline & & & & & & & & & 0.2 & & 0.89194 & 0.47689 & 1.01249 \\
\hline & & & & & & & & & 0.4 & & 0.89215 & 0.47672 & 1.17366 \\
\hline & & & & & & & & & 0.6 & & 0.89215 & 0.47672 & 1.34053 \\
\hline & & & & & & & & & & 1 & 1.47589 & 0.07142 & 0.57956 \\
\hline & & & & & & & & & & 2 & 0.89215 & 0.47672 & 1.34053 \\
\hline & & & & & & & & & & 3 & 0.34586 & 0.88690 & 2.10581 \\
\hline
\end{tabular}

Table 3. Values of $-\theta^{\prime}(0)$ for $\operatorname{Pr}=10$.

\begin{tabular}{cccccc}
\hline $\boldsymbol{M}$ & $\boldsymbol{N} \boldsymbol{t}$ & $\boldsymbol{N} \boldsymbol{b}$ & $\begin{array}{c}-\boldsymbol{\theta}^{\prime}(\mathbf{0}) \\
\text { base fluid }\end{array}$ & $\begin{array}{c}-\boldsymbol{\theta}^{\prime}(\mathbf{0}) \\
\text { nanofluid }\end{array}$ & Relative\% \\
\hline 0.1 & 0.1 & 0.1 & 1.87976 & 2.29010 & $15.68 \%$ \\
0.3 & - & - & 1.79877 & 2.19766 & $14.96 \%$ \\
0.5 & - & - & 1.68542 & 1.84631 & $9.11 \%$ \\
& & & & & \\
0.7 & - & - & 1.18450 & 1.21349 & $2.41 \%$ \\
1 & - & - & 0.94877 & 1.03470 & $8.66 \%$ \\
\hline
\end{tabular}

movement and slows down the fluid velocity. The impact of $\beta$ on velocity field $f^{\prime}(\eta)$ is presented in Figure 4. As noticed, both flow and thickness of boundary layer increased upon increasing the $\beta$ values. Physically, in clinical chemistry, skin turgor is the skin elasticity. It is the ability of skin to change shape and return to normal. Skin turgor is the symptom of dehydration. Since $\beta$ is highly dependent on the stretching rate $a$, an increase in $\beta$ would increase the fluid motion in the boundary-layer close to the surface. Consequently, it rises the velocity and thickness of the boundary layer. Figure 5 reveals the impact of values of the relaxation over retardation time $\lambda$ on the flow field. The velocity $f^{\prime}(\eta)$ was reduced as $\lambda$ increased. The thickness of the velocity boundary layer increased as $\lambda$ rose. As observed, the boundary layer (momentum) diminishes in greater values of $\lambda$. Since $\lambda$ is oppositely related to the time of retardation of the non-Newtonian fluid, upon raising $\lambda$, the time of retardation and subsequently the fluid flow decreases.

Figure 6 shows the influence of $P r$ on the thermal profile $\theta(\eta)$. Following an increase in $\operatorname{Pr}$ value, the temperature field decreased, because at the larger value of $P r$, the thermal diffusivity of the liquid is 


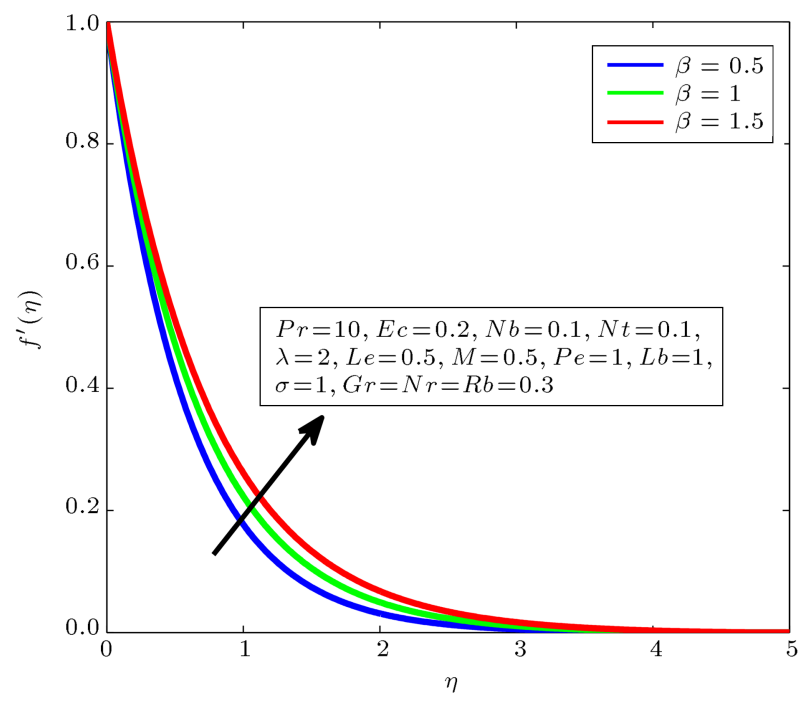

Figure 4. Influence of $\beta$ on $f^{\prime}(\eta)$.

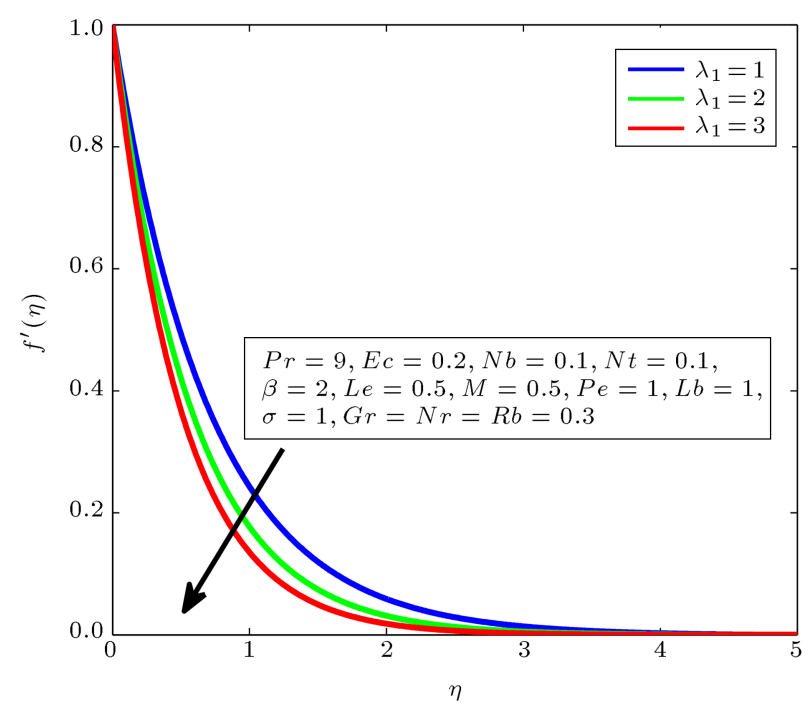

Figure 5. Influence of $\lambda$ on $f^{\prime}(\eta)$.

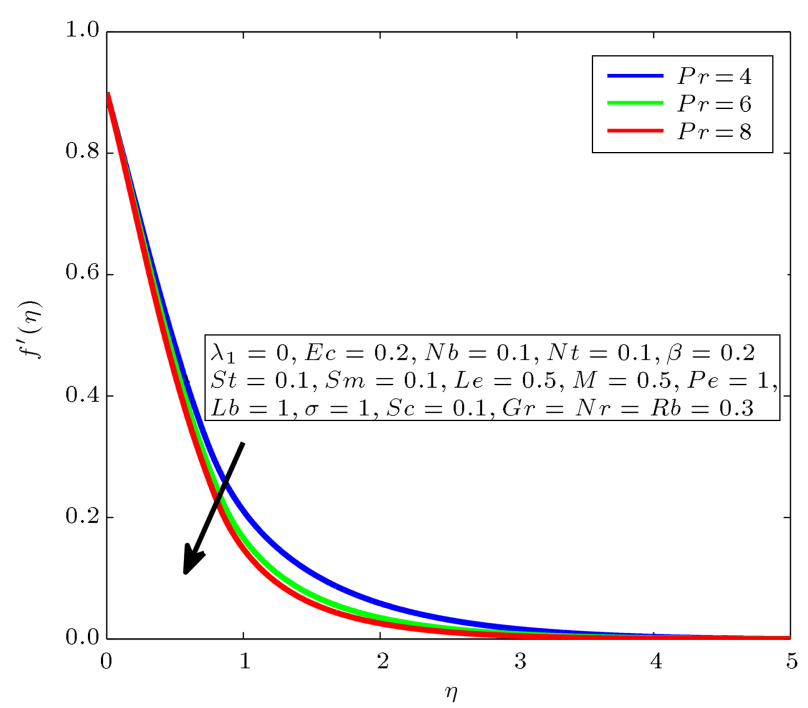

Figure 6. Influence of $\operatorname{Pr}$ on $\theta(\eta)$.

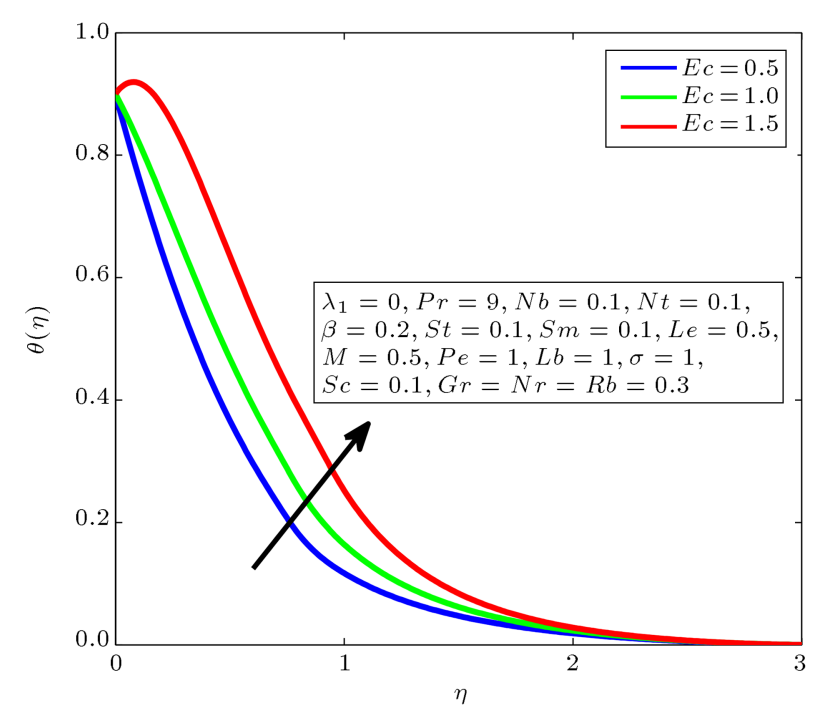

Figure 7. Influence of $E c$ on $\theta(\eta)$.

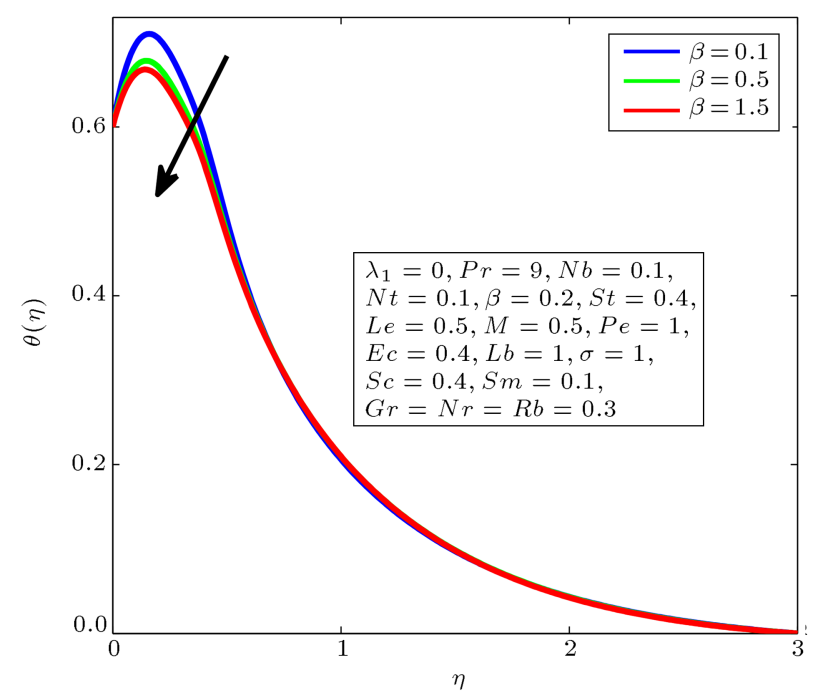

Figure 8. Influence of $\beta$ on $\theta(\eta)$.

reduced. Physically, Prandtl number controls the relative thickness of the momentum and thermal boundary layers. Small Pr value indicates that heat diffuses more quickly than the velocity (momentum). Thus, in the case of liquid metals, the thermal boundary layer is much thicker than the velocity boundary layer. Consequently, the temperature profile of the fluid is reduced. Figure 7 exhibits the sway of Eckert number $E c$ on the fluid temperature $\theta$. Figure 7 shows that an increase in the value of $E c$ enhances the thermal field $\theta$, simply because heat is accumulated in the fluid caused by a frictional heating. Further close to the surface, thermal profile overshoots with an increment in $E c$. From Figure 8, it is obvious that temperature declines at rising values of $\beta$. In a physical sense, $\beta$ is directly related to the time of retardation; therefore, the retardation time is enhanced whenever $\beta$ increases. This rise in retardation time contributes to reduction of 


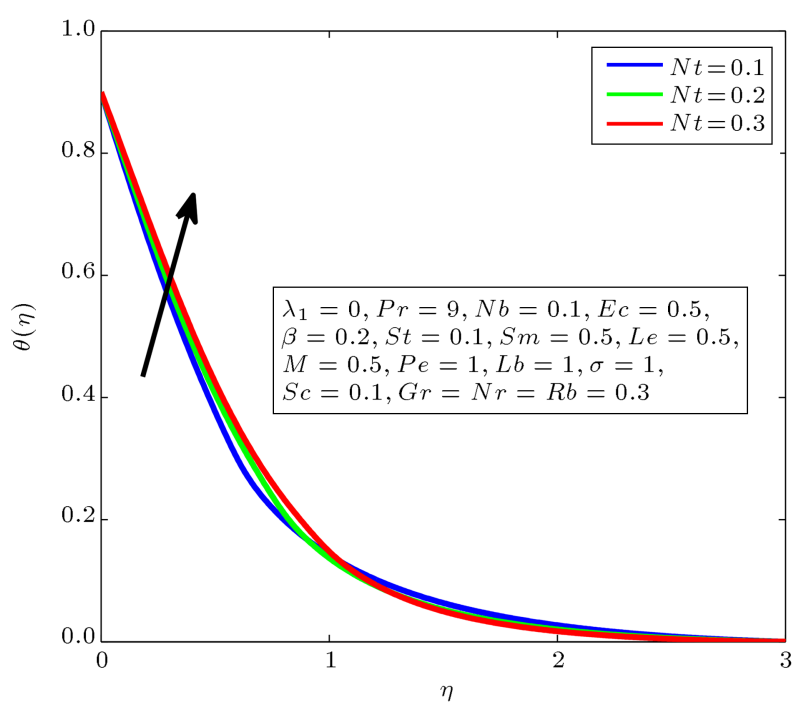

Figure 9. Influence of $N t$ on $\theta(\eta)$.

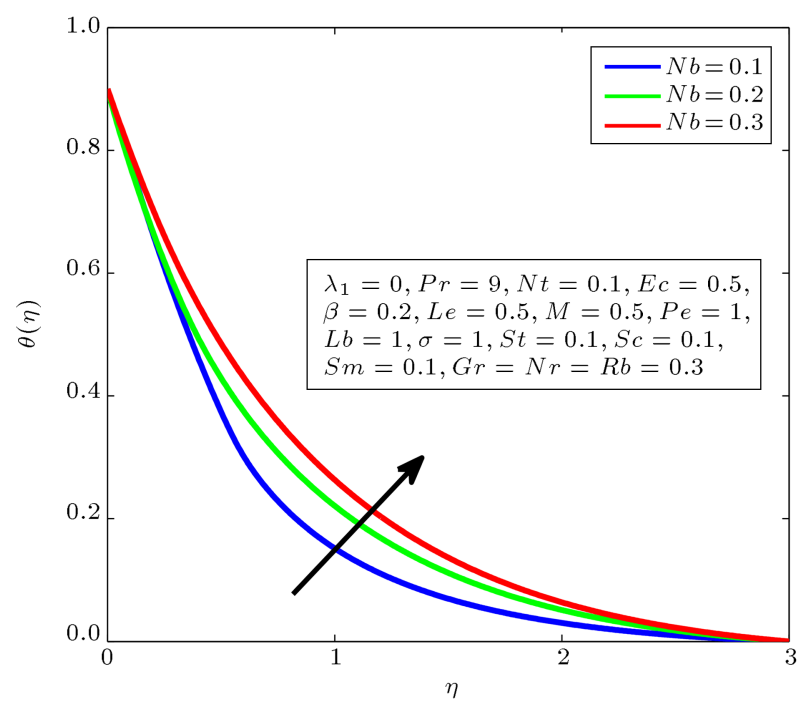

Figure 10. Influence of $N b$ on $\theta(\eta)$.

the temperature together with the thickness of thermal boundary layer.

Through Figure 9, it is noted that for escalating values of $N t$, the temperature profile exhibits an increasing trend. Physically, $N t$ enhances the density of the thermal boundary layer, due to which the temperature profile increases. Figure 10 shows the impact of the Brownian motion parameter $N b$ on $\theta$. The temperature increases with the escalating values of $N b$. It is generally because a rise in $N b$ strengthens the random motion of the fluid particles as a result of which excess heat is generated. Therefore, the temperature of the fluid increases. Figure 11 highlights the impact of the thermal stratification parameter $S t$ on $\theta(\eta)$. In general, in case of $S t$ rise, the difference in the temperatures of heated sheet and ambient is reduced. Hence, the fluid temperature declines.

Figure 12 reveals the effect of $\mathrm{Nb}$ on the con-

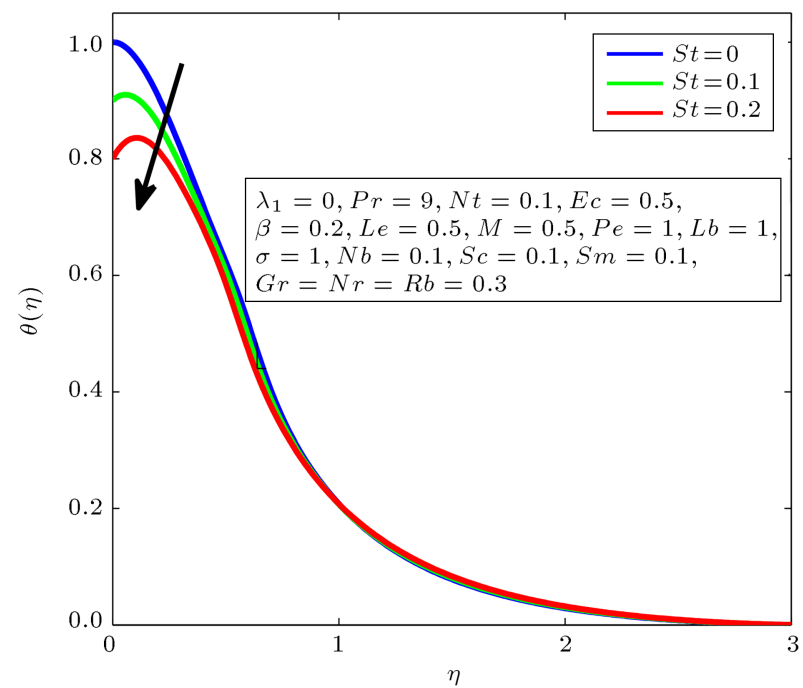

Figure 11. Influence of $S t$ on $\theta(\eta)$.

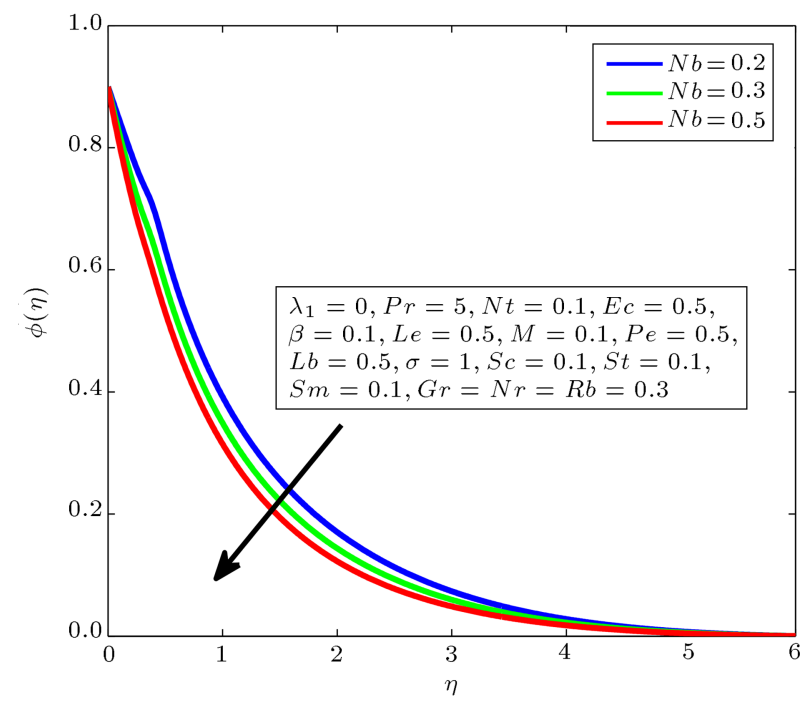

Figure 12. Influence of $N b$ on $\phi(\eta)$.

centration of nanoparticles $\phi(\eta)$. It is perceived that an increase in the values of $N b$ reduces the concentration profile $\phi(\eta)$. Generally, an increase in the value of Brownian motion parameter speeds up the collision among the fluid particles, resulting in excess heat. As a result, the concentration profile $\phi(\eta)$ is reduced. Figure 13 suggests the impact of $N t$ on the mass fraction function $\phi(\eta)$. It can be noted that an intensification in $N t$ boosts up the concentration profile. It is manifested that a larger value of $N t$ results in an enlargement of the thermophoresis force within the boundary-layer region that accelerates the mass fraction of the nanoparticles. Figure 14 presents the concentration profile $\phi(\eta)$ for different values of Le. The concentration of nanoparticles declines for the growing values of the Lewis number Le. Because the ratio of thermal diffusivity over mass diffusivity is defined as the Lewis number, the larger values of $L e$ 


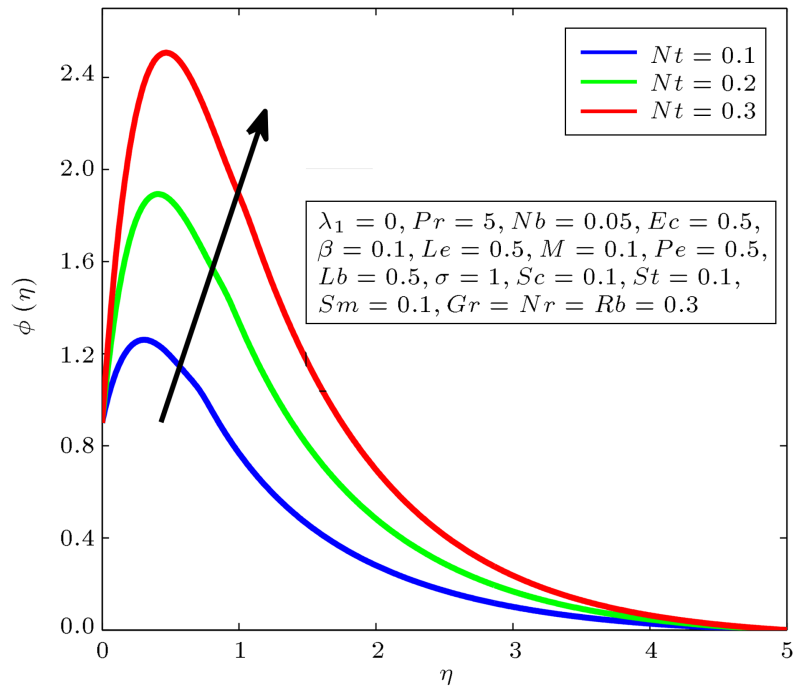

Figure 13. Influence of $N t$ on $\phi(\eta)$.

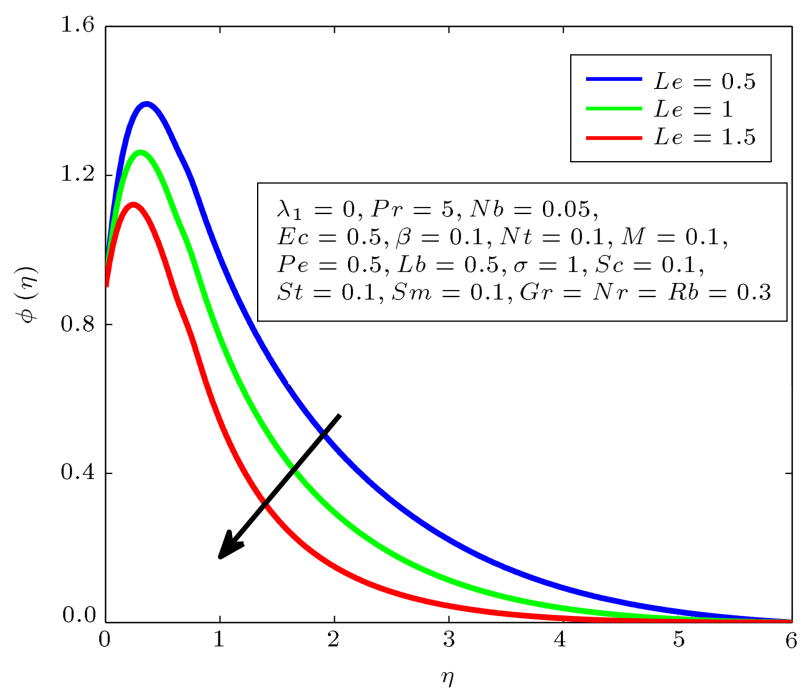

Figure 14. Influence of Le on $\phi(\eta)$.

reduce the mass fraction function. Consequently, the concentration profile is reduced by enhancing the value of $L e$. The effect of the solutal stratification parameter $S c$ on the mass fraction function $\phi(\eta)$ is reflected in Figure 15. It can be very clearly viewed that $\phi(\eta)$ shows a diminishing behavior in the case of increase in the value of the solutal stratification parameter $S c$.

Figure 16 shows the behavior of the gyro-tactic microorganism density profile $\chi(\eta)$ for different values of the Peclet number $P e$. Peclet number is defined by advective over diffusive transport rate. According to this figure, a rise in $P e$ causes a decrement in the motile microorganism density profile, because a rise in $P e$ enhances the motion of the fluid particles that induces a decline in the thickness of gyro-tactic micro-organism. Figure 17 is sketched to highlight the effect of the bio-convection number $L b$ on the motile density. Conducted analysis illustrates that in the large

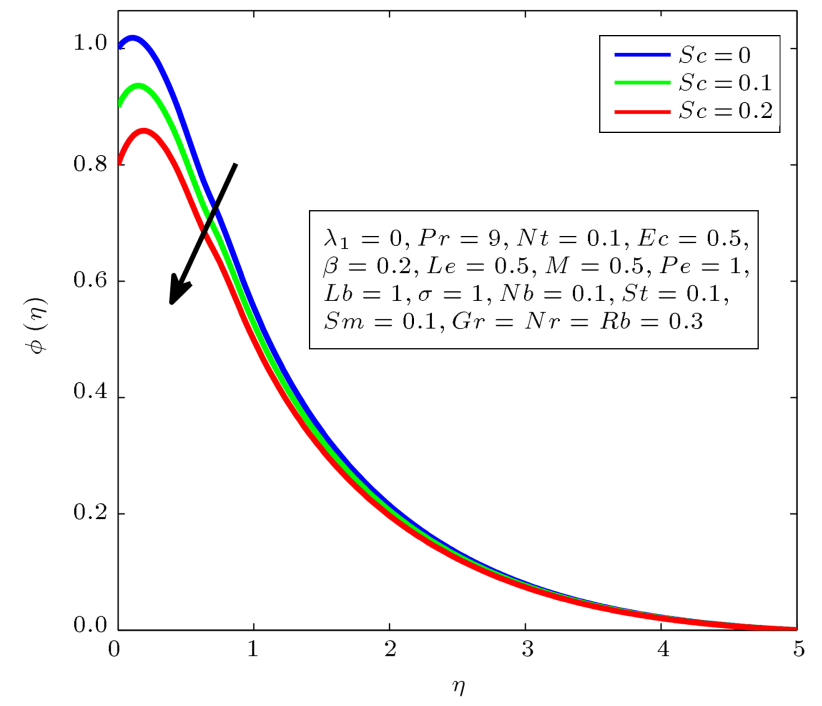

Figure 15. Influence of $S c$ on $\phi(\eta)$.

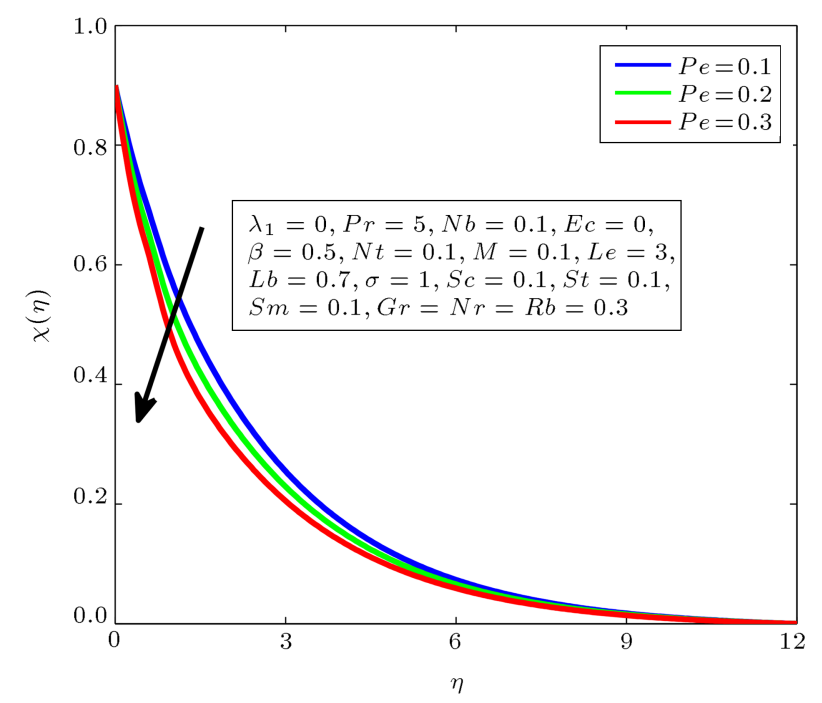

Figure 16. Influence of $P e$ on $\chi(\eta)$.

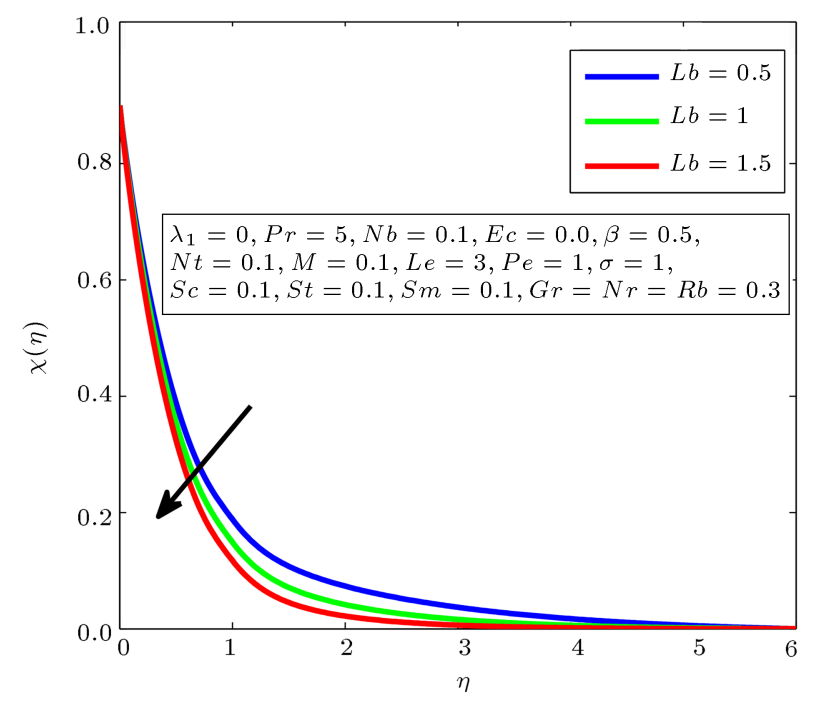

Figure 17. Influence of $L b$ on $\chi(\eta)$. 


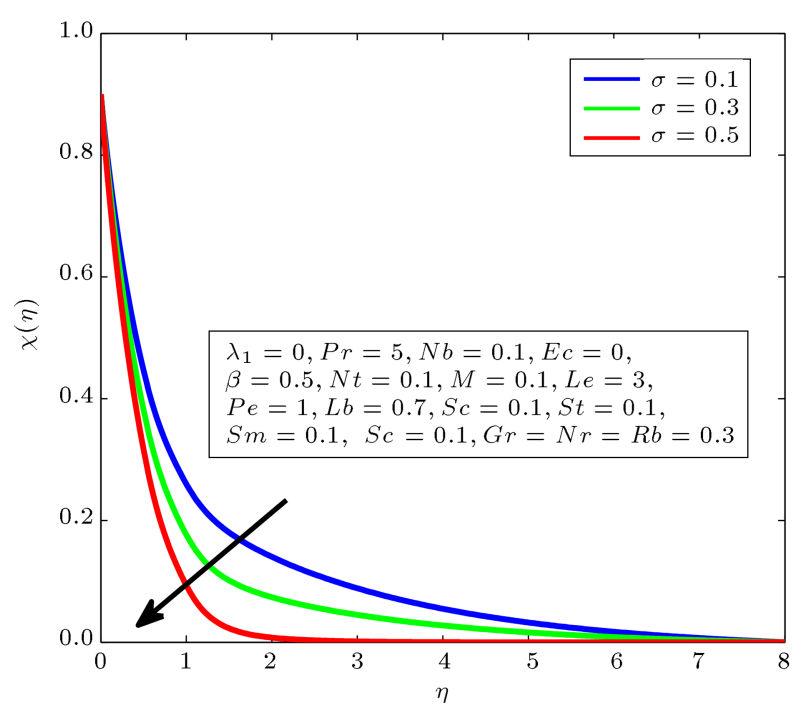

Figure 18. Influence of $\sigma$ on $\chi(\eta)$.

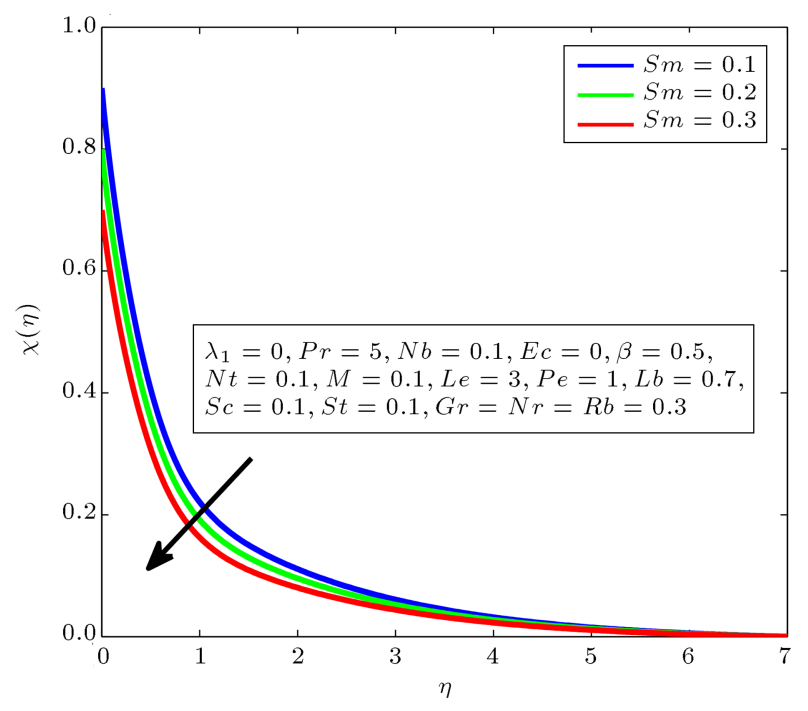

Figure 19. Influence of $S m$ on $\chi(\eta)$.

value of the bio-convection number, the diffusivity of micro-organisms is reduced as a result of which the density of the gyro-tactic micro-organisms is seen to deteriorate. Figure 18 shows that the concentration of microorganisms $\chi(\eta)$ suffers a decreasing trend for an increment in the concentration difference parameter $\sigma$ associated with the micro-organism. According to Figure 19, an enhancement in $S m$ considerably lessens the concentration difference of the micro-organisms between the sheet and far from the sheet and, therefore, a reduction in the density profile is observed.

Figure 20 presents the behavior of the local drag coefficient $f^{\prime \prime}(0)$ at various values of $\beta$ and $M$. According to the figure, upon increasing the value of the Deborah number, the skin friction coefficient experience an enhancement. Additionally, the skin friction coefficient exhibits a decreasing behavior at the increased values of the magnetic parameter $M$.

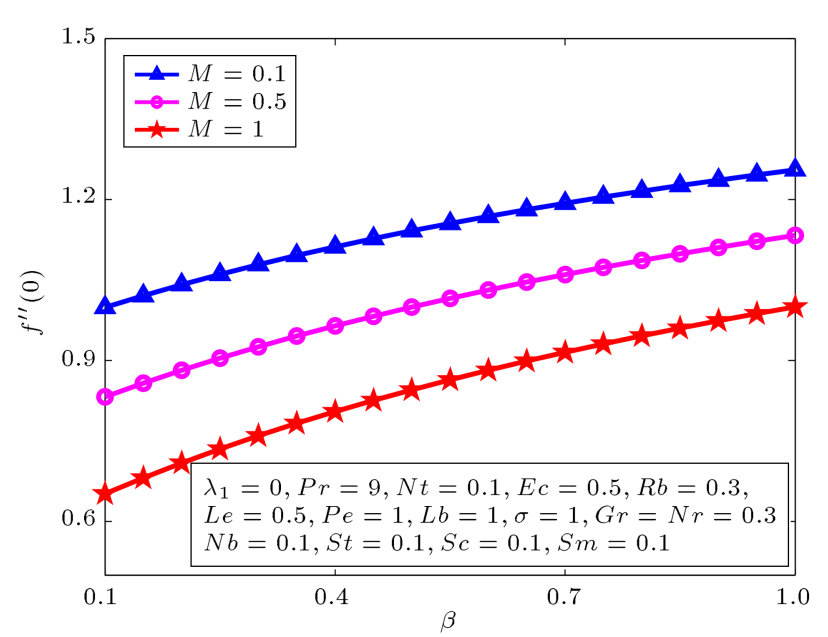

Figure 20. Influence of $\beta$ on $f^{\prime \prime}(0)$ for different values of $M$.

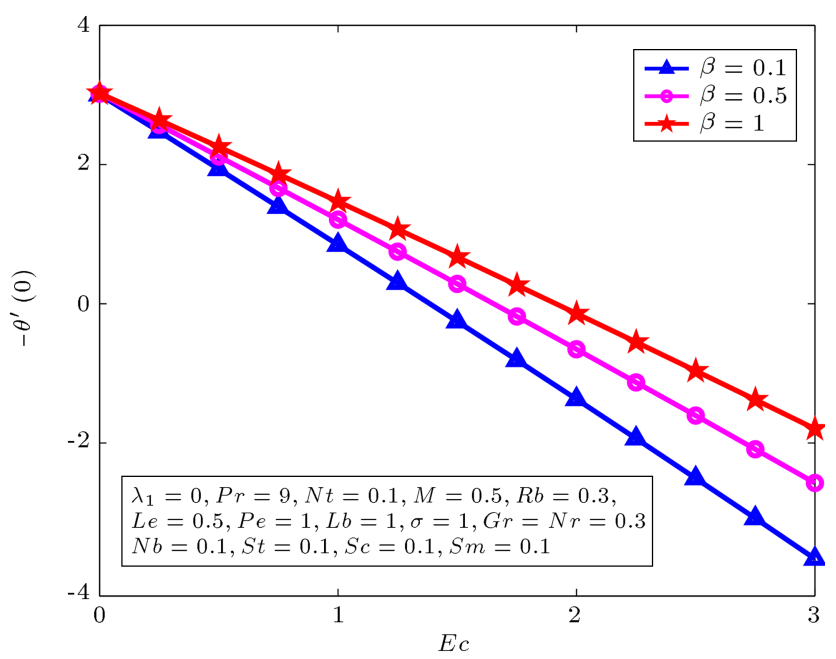

Figure 21. Influence of $E c$ on $-\theta^{\prime}(0)$ for different values of $\beta$.

Figure 21 exhibits the heat transfer rate $-\theta^{\prime}(0)$ affected by the Eckert number $E c$ for a variety of values of $\beta$. A rise in $\beta$ boosts the heat transfer rate and it is reduced at larger values of $E c$. Figure 22 shows the effect of the parameters $N b$ and $N t$ on the mass transfer rate $-\phi^{\prime}(0)$. It has been concluded that $-\phi^{\prime}(0)$ increases with an augmentation in $N b$, while it decreases at the larger value of $N t$. Figure 23 describes that the mass transfer rate $-\phi^{\prime}(0)$ is enhanced with a rise in $N b$ and Le. The impact of $L b$ on the local micro-organism transfer rate $-\phi^{\prime}(0)$ for a variety of values of $P e$ is presented in Figure 24. It can be noticed that $-\chi^{\prime}(0)$ is enhanced with an increase in $L b$, because the convection of the motile microorganism is amplified with a rise in $L b$. The local micro-organism transfer rate $-\chi^{\prime}(0)$ is reduced for the rising values of the bioconvection Peclet number Pe. From Figure 25, it is found that the local micro-organism transfer rate $-\chi^{\prime}(0)$ is reduced for the greater micro-organisms 


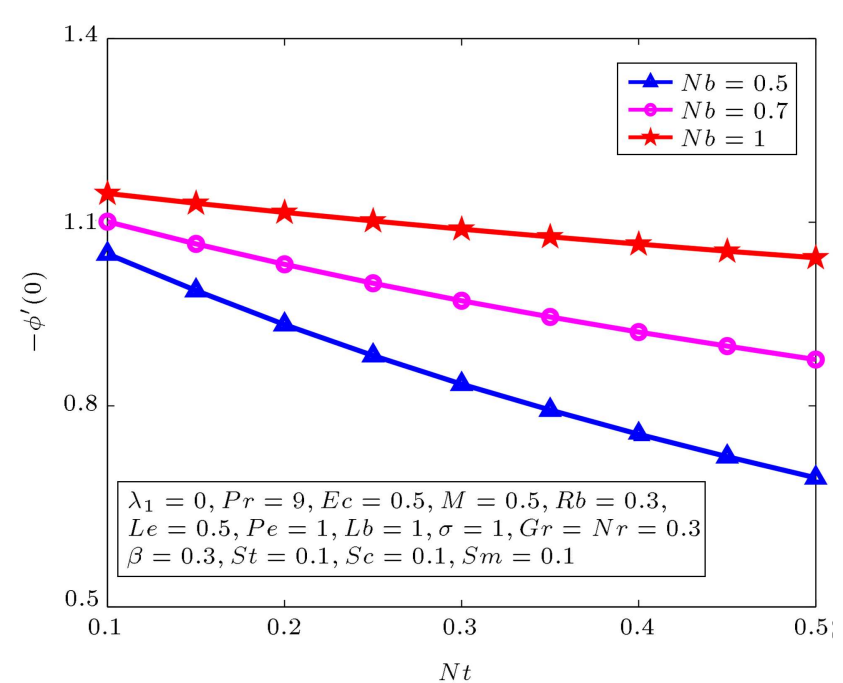

Figure 22. Influence of $N t$ on $-\phi^{\prime}(0)$ for different values of $N b$.

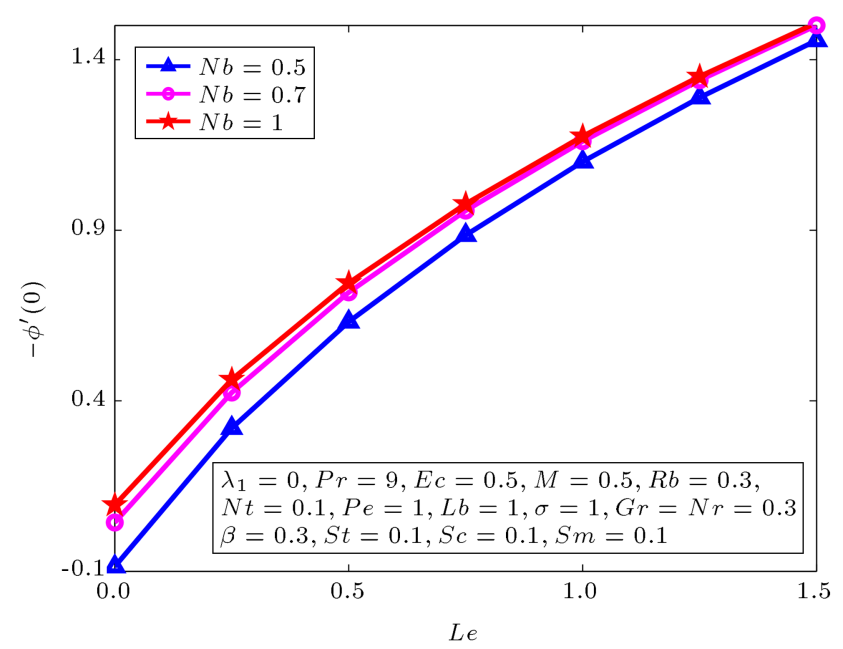

Figure 23. Influence of $L e$ on $-\phi^{\prime}(0)$ for different values of $N b$.

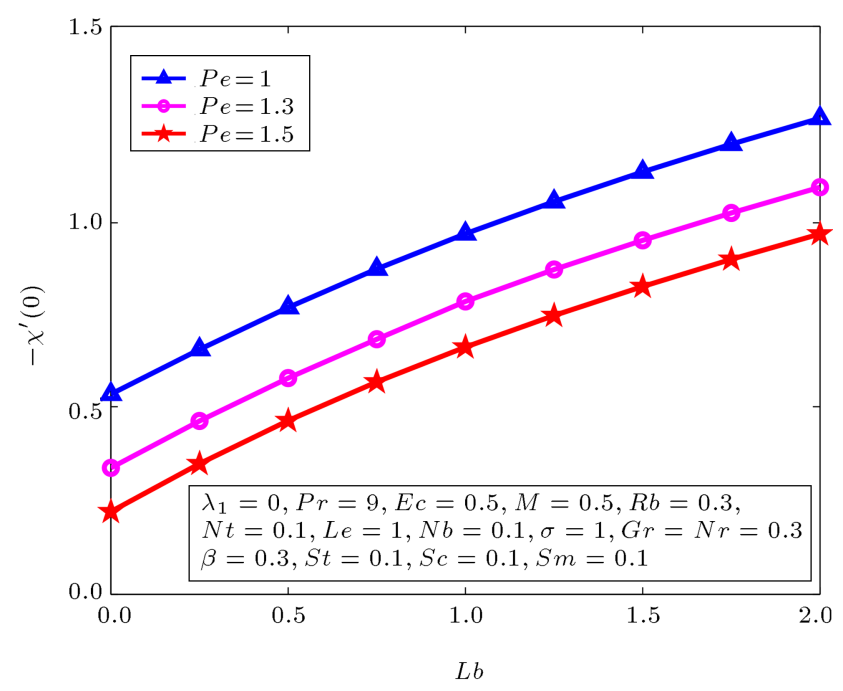

Figure 24. Variation of $-\chi^{\prime}(0)$ against $L b$ for different values of $P e$.

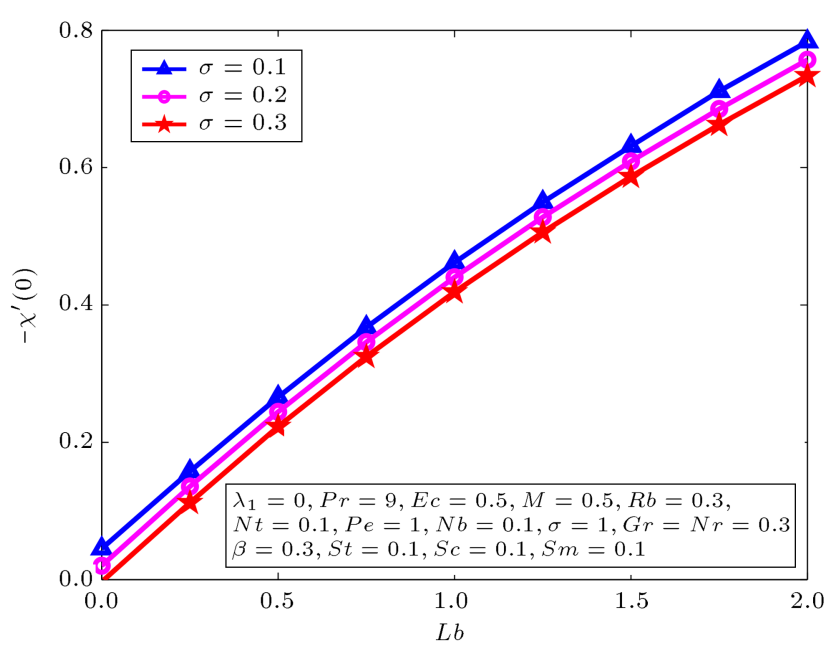

Figure 25. Variation of $-\chi^{\prime}(0)$ against $L b$ for different values of $\sigma$.

concentration difference parameter $\sigma$; however, the greater values of $\mathrm{Lb}$ are observed to cause an increment in the $-\chi^{\prime}(0)$.

\section{Concluding remarks}

This paper aims to explore the conduct of the stratified MHD Jeffrey nanofluid flow subjected to an expandable surface. Heat and mass transfer analysis was carried out with the inclusion of distinguished effects. Heat transport has different industrial applications like extrusions, steam generators, molding, gas processing, refineries, etc. Mass transport has different applications in industry like adsorbers in carbon beds, scrubbers or stripping, and liquid-liquid extraction. Mass transfer is often coupled to additional transport processes, for instance in industrial cooling towers. The main outcomes of this research are listed below:

- The concentration of the motile micro-organisms was a reducing function of the bioconvection Lewis number, the bioconvection Peclet number, and concentration difference for micro-organisms;

- An increase in the Deborah number was a cause for the flow field though the magnetic parameter to a decline;

- The velocity and the locals Nusselt number were the diminishing functions of the magnetic parameter;

- The drag coefficient and the local heat transfer rate increased with an increase in the Deborah number;

- Sherwood number was enhanced with a growth in thermophoresis parameter, but suffered a decrement due to the Brownian motion parameter;

- The motile density number of micro-organisms was enhanced with a rise in the bioconvection Lewis number; however, it is diminished by augmenting the Peclet number; 
- The thermal efficiency of nanofluid over conventional fluid was seen with a minimum of $2.41 \%$ and maximum of $15.68 \%$;

- The local Nusselt number and the local density number rose with an escalation in the thermophoresis as well as the Brownian motion parameters;

- The thermal, mass, and motile density stratification parameters decreased the temperature, concentration of nanoparticles, and motile density of the micro-organism's profiles;

- For a fixed volume fraction of nanoparticles, the nanofluid is a better heat transport agent than the conventional fluid.

\section{Acknowledgements}

The authors would like to express their sincere gratitude to the reviewers and the editor for their careful reading and helpful remarks which helped enhance the overall quality of this paper.

\section{Nomenclature}

$u, v \quad$ velocity components

$x, y \quad$ Cartesian coordinate

$C \quad$ Concentration off nanoparticles

$C_{w} \quad$ Wall concentration of nanoparticles

$C_{f} \quad$ Skin friction

$D_{B} \quad$ Brownian diffusion coefficient

$D m \quad$ Diffusivity of microorganisms

$D_{T} \quad$ Thermophoretic diffusion coefficient

$f \quad$ Dimensionless stream function

$W_{c} \quad$ Constant maximum cell swimming speed

$B_{0} \quad$ Magnetic field intensity

$q_{w} \quad$ Wall heat flux

$L b \quad$ Bioconvection Lewis number

Le Lewis number

$C_{0} \quad$ Reference concentration

$T_{0} \quad$ Reference temperatures

$N_{0} \quad$ Reference density of micro-organisms

$N n_{x} \quad$ Local density number

Nt Thermophoresis parameter

$N u_{x} \quad$ Local Nusselt number

$\operatorname{Pr} \quad$ Prandtl numbers

$P e \quad$ Bioconvection Peclet number

$T \quad$ Temperature of the fluid

$q_{m} \quad$ Wall mass flux

$q_{n} \quad$ Wall motile microorganisms flux

$\tau_{w} \quad$ Wall shear stress $c$

$\sigma$

$\tau$

Gr

$R b$

$\gamma^{*}$

$N$

M

$b$

$c_{p}$

$S h_{x}$

$N_{w}$

$T_{\infty}$

$\lambda$

$\lambda_{1}$

$k$

$\psi$

$\eta$

$\theta$

$\beta$

$\sigma_{f}$

$\sigma_{p}$

$\mu$

$\alpha$

$\phi$

$T_{w}$

$\chi$

$N_{\infty}$

Ec

St

Sc

$S m$

$U_{w}$

$q_{w}$

$c$

$N r$

$\beta^{*}$

$\rho_{m}$
Stretching rate

Bioconvection concentration difference

Heat capacity ratio

Mixed convection parameter

Bioconvection Rayleigh number

Average volume of microorganisms

Concentration of micro-organism

Magnetic parameter

Chemotaxis constant

Specific heat

Local Sherwood number

Wall density of micro-organism

Ambient temperature

Relaxation to retardation time

Retardation time

Thermal conductivity

Stream function

Similarity variable

Dimensionless temperature

Deborah number

Electrical conductivity

Density of nanoparticles

Viscosity of fluid

Thermal diffusivity

Dimensionless concentration

Wall temperature

Dimensionless density of microorganism

Ambient density of micro-organism

Eckert number

Thermals stratification parameter

Mass stratification parameter

Motile density stratification parameter

Stretching velocity along $x$-axis

Wall heat flux

Positive constant

Buoyancy ratio parameter

Volume expansion coefficient

Density of microorganism

\section{References}

1. Stewart, T.L. and Fogler, H.S. "Biomass plug development and propagation in porous media", Biotechnology and Bioengineering, 72(3), pp. 353-363 (2001).

2. Polyansky, O. and Vladimir, R. "Fluid convection in sediment-hosted reservoirs due to thermal action of dikes and sills", Russian Geology and Geophysics, 43, pp. 25-39 (2002). 
3. Kuznetsov, A.V. "Bio-thermal convection induced by two different species of microorganisms", International Communications in Heat and Mass Transfer, 38(5), pp. 548-553 (2011).

4. Khan, W.A. and Makinde, O.D. "MHD nanofluid bioconvection due to gyrotactic microorganisms over a convectively heat stretching sheet", International Journal of Thermal Sciences, 81, pp. 118-124 (2014).

5. Makinde, O.D. and Animasaun, I.L. "Bioconvection in MHD nanofluid flow with nonlinear thermal radiation and quartic autocatalysis chemical reaction past an upper surface of a paraboloid of revolution", International Journal of Thermal Sciences, 109, pp. 159-171 (2016).

6. Xun, S., Zhao, J., Zheng, L., et al. "Bioconvection in rotating system immersed in nanofluid with temperature dependent viscosity and thermal conductivity", International Journal of Heat and Mass Transfer, 111, pp. 1001-1006 (2017).

7. Mosayebidorcheh, S., Tahavori, M.A., Mosayebidorcheh, T., et al. "Analysis of nanobioconvection flow containing both nanoparticles and gyrotactic microorganisms in a horizontal channel using modified least square method", Journal of Molecular Liquids, 227, pp. 356-365 (2017).

8. Maxwell, J.C., A Treatise on Electricity and Magnetism, Clarendon Press Oxford UK (1873).

9. Choi, S.U.S. and Eastman, J.A. "Enhancing thermal conductivity of fluids with nanoparticles", $A S M E$ Publications-Fed, 231, pp. 99-106 (1995).

10. Wong, K.V. and Leon, O.D. "Applications of nanofluids: Current and future", Advances in Mechanical Engineering, 2, pp. 1-11 (2010).

11. Kostic, M., Critical Issues in Nanofluids Research and Application Potentials, Nova Science Publisher (2013).

12. Buongiorno, J. "Convective transport in nanofluids", Journal of Heat Transfer, 128, pp. 240-250 (2006).

13. Sheikholeslami, M., Farshad, S.A., Said, Z., et al. "Analyzing entropy and thermal behavior of nanomaterial through solar collector involving new tapes", International Communications in Heat and Mass Transfer, 123, p. 105190 (2021).

14. Shahrestani, I., Maleki, A., Shadloo, S., et al. "Numerical investigation of forced convective heat transfer and performance evaluation criterion of $\mathrm{Al}_{2} \mathrm{O}_{3} /$ water nanofluid flow inside an axisymmetric microchannel", Symmetry, 12(1), p. 120 (2020).

15. Lahmar, S., Kezzar, M., Eid, R., et al. "Heat transfer of squeezing unsteady nanofluid flow under the effects of an inclined magnetic field and variable thermal conductivity", Physica A: Statistical Mechanics and Its Applications, 540, pp. 123-138 (2020).

16. Sheikholeslami, M. and Farshad, S.A. "Investigation of solar collector system with turbulator considering hybrid nanoparticles", Renewable Energy, 171, pp. 1128-1158 (2021).
17. Ibrahim, S.M., Lorenzini, G., Kumar P., et al. "Influence of chemical reaction and heat source on dissipative MHD mixed convection flow of a Casson nanofluid over a nonlinear permeable stretching sheet", International Journal of Heat and Mass Transfer, 111, pp. 346-355 (2017).

18. Sheikholeslami, M., Farshad, S.A., Ebrahimpour, Z., et al. "Recent progress on flat plate solar collectors and photovoltaic systems in the presence of nanofluid: A review", Journal of Cleaner Production, 293, pp. 126119 (2021).

19. Jeffreys, H., The Earth, 4th edition, Cambridge University Press, London (1929).

20. Saif, R.S., Muhammad, T., Sadia, H., et al. "Hydromagnetic flow of Jeffrey nanofluid due to a curved stretching surface", Physica A: Statistical Mechanics and Its Applications, 551, p. 124060 (2020).

21. Narayana, P.V., Babu, D., Venkateswarlu, B., et al. "Soret and Dufour effects on MHD radiative heat and mass transfer flow of a Jeffrey fluid over a stretching sheet", Frontiers in Heat and Mass Transfer, 8, pp. 1-9 (2017).

22. Jena, S., Mishra, S.R., and Dash, G.C. "Chemical reaction effect on MHD Jeffery fluid flow over a stretching sheet through porous media with heat generation/absorption", International Journal of Applied and Computational Mathematics, 3(2), pp. 1225-1238 (2017).

23. Sreelakshmi, K., Sarojamma, G., and Murthy, J.V. "Homotopy analysis of an unsteady flow heat transfer of a Jeffrey nanofluid over a radially stretching convective surface", Journal of Nanofluids, 7(1), pp. 62-71 (2018).

24. Ahmad, K., Hanouf, Z., and Ishak, A. "Mixed convection Jeffrey fluid flow over an exponentially stretching sheet with magnetohydrodynamic effect", $A I P A d$ vances, 6(3), p. 035024 (2016).

25. Bearon, R.N. and Grunbaum, D. "Bioconvection in a stratified environment: experiments and theory", Physics of Fluids, 18(12), p. 127102 (2006).

26. Kameswaran, K., Vasu, B., Murthy, N., et al. "Mixed convection from a wavy surface embedded in a thermally stratified nanofluid saturated porous medium with non-linear boussinesq approximation", International Communications in Heat and Mass Transfer, 77, pp. 78-86 (2016).

27. Madhu, V. and Reddy, S. "Influence of thermal stratification on MHD heat transfer flow over an exponentially stretching surface", International Journal of Scientific Research in Science and Technology, 4, pp. 832-838 (2018).

28. Sakiadis, B.C. "Boundary layer behavior on continuous solid flat surfaces", American Institute of Chemical Engineers Journal, 7, pp. 26-28 (1961).

29. Crane, L. "Flow past a stretching plate", Journal of Applied Mathematics and Physics, 21, pp. 645-647 (1970). 
30. Tsou, F.K., Sparrow, E.M., and Goldstein, R.J. "Flow and heat transfer in the boundary layer on a continuous moving surface", International Journal of Heat and Mass Transfer, 10, pp. 219-235 (1967).

31. Mehmood, Y., Sagheer M., and Hussain, S. "MHD oblique stagnation point flow of nanofluid over a convective stretching surface", Journal of Computational and Theoretical Nanoscience, 14(4), pp. 1724-1734 (2017).

32. Saif, R.S., Muhammad, T., Sadia, H., et al. "Boundary layer flow due to a nonlinear stretching curved surface with convective boundary condition and homogeneousheterogeneous reactions", Physica A: Statistical Mechanics and Its Applications, 551, pp. 123996 (2020).

33. Venkateswarlu, B. and Narayana, P.V. "Influence of variable thermal conductivity on MHD Casson fluid flow over a stretching sheet with viscous dissipation, Soret and Dufour effects", Frontiers in Heat and Mass Transfer, 7, p. 16 (2016).

34. Saif, R.S., Muhammad, T., and Sadia, H. "Significance of inclined magnetic field in Darcy Forchheimer flow with variable porosity and thermal conductivity", Physica A: Statistical Mechanics and Its Applications, 551, pp. 124067 (2020).

35. Sarpakaya, T. "Flow of non-Newtonian fluids in a magnetic field", American Institute of Chemical Engineers, 7(2), pp. 324-328 (1961).

36. Elbashbeshy, E.M.A., Emam, T.G., and Abdelgaber, K.M. "Effects of thermal radiation and magnetic field on unsteady mixed convection flow and heat transfer over an exponentially stretching surface with suction in the presence of internal heat generation/ absorption", Journal of the Egyptian Mathematical Society, 20, pp. 215-222 (2012).

37. Animasaun, I.L. "Effects of thermophoresis, variable viscosity and thermal conductivity on free convective heat and mass transfer of non-Darcian MHD dissipative Casson fluid flow with suction and nth order of chemical reaction", Journal of the Nigerian Mathematical Society, 30, pp. 187-195 (2013).

38. Nayak, M.K., Akbar, N.S., Pandey, V.S., et al. "3D free convective MHD flow of nanofluid over permeable linear stretching sheet with thermal radiation", Powder Technology, 315, pp. 205-215 (2017).

39. Abel, M.S., Kumar, K., and Ravikumara, R. "MHD flow and heat transfer with effects of buoyancy, viscous and Joule dissipation over a nonlinear vertical stretching porous sheet with partial slip", Engineering, 3, pp. 285-291 (2011).

40. Kishan, N. and Deepa, G. "Viscous dissipation effects on stagnation point flow and heat transfer of a micropolar fluid with uniform suction or blowing", Advances in Applied Science Research, 3, pp. 430-439 (2012).

41. Alim, M.A., Alam, M.M., Mamun, A.A., et al. "Combined effect of viscous dissipation and Joule heating on the coupling of conduction and free convection along a vertical flat plate", International Communications in Heat and Mass Transfer, 35, pp. 338-346 (2008).
42. Ferdows, M., Afify, A.A., and Tzirtzilakis, E.E. "Hall current and viscous dissipation effects on boundary layer flow of heat transfer past a stretching sheet", International Journal of Applied and Computational Mathematics, 3(4) pp. 1-17 (2017).

43. Selcuk, S. and Ziyaddin, R. "Impact of electrical and magnetic field on cooling process of liquid metal duct magnetohydrodynamic flow", Thermal Science, 22, pp. 263-271 (2018).

44. Selimli, S., Recebli, Z., and Arcaklioglu, E. "Combined effects of magnetic and electrical field on the hydrodynamic and thermophysical parameters of magnetoviscous fluid flow", International Journal of Heat and Mass Transfer, 86, pp. 426-432 (2015).

45. Recebli, Z., Selimli, S., and Ozkaymak, M. "Theoretical analyses of immiscible MHD pipe flow", International Journal of Hydrogen Energy, 40(44), pp. 1536515373 (2015).

46. Selimli, S., Recebli, Z., and Arcaklioglu, E. "MHD numerical analyses of hydrodynamically developing laminar liquid lithium duct flow", International Journal of Hydrogen Energy, 40(44), pp. 15358-15364 (2015).

47. Rundora, L. and Makinde, O.D. "Analysis of unsteady MHD reactive flow of non-Newtonian fluid through a porous saturated medium with asymmetric boundary conditions", Iranian Journal of Science and Technology, Transactions of Mechanical Engineering, 40(3), pp. 189-201 (2016).

48. Makinde, O.D., Eegunjobi, A.S., Shehla, T., et al. "Thermodynamics analysis of variable viscosity hydromagnetic Couette flow in a rotating system with Hall effects", Entropy, 17(11), pp. 7811-7826 (2015).

49. Hooman, K., Li, J., and Dahari, M. "Thermal dispersion effects on forced convection in a porous-saturated pipe", Thermal Science and Engineering Progress, 2, pp. 64-70 (2017).

50. Ijaz, M., Waqas, M., Hayat, T., et al. "Behavior of stratification phenomenon in flow of Maxwell nanomaterial with motile gyrotactic microorganisms in the presence of magnetic field", International Journal of Mechanical Sciences, 131-132, pp. 426-434 (2017).

51. Cebeci, T. and Bradshaw, P., Physical and Computational Aspects of Convective Heat Transfer, Springer New York (1988).

52. Keller, H.B., Numerical Methods for Two-Point Boundary Value Problems, Dover Publications New York (1992).

53. Ishak, A., Nazar, R., and Pop, I. "Hydromagnetic flow and heat transfer adjacent to a stretching vertical sheet", Heat and Mass Transfer, 44(8), p. 921 (2008).

54. Pal, D. and Mondal, H. "Hydromagnetic non-Darcy flow and heat transfer over a stretching sheet in the presence of thermal radiation and Ohmic dissipation", Communications in Nonlinear Science and Numerical Simulation, 15(5), pp. 1197-1209 (2010). 


\section{Biographies}

Faisal Shahzad received his $\mathrm{PhD}$ degree in Applied Mathematics from Capital University of Science and Technology (CUST). His research interests are nanofluid, CFD, simulation, mass transport, heat transfer, magnetrohydrodynamics, numerical and analytical methods, and hybrid nanofluids.

Muhammad Sagheer received his $\mathrm{PhD}$ degree in 2005 from the University of Sussex, England. He joined NUST Institute of Information Technology (SEECS) in 2006 for a year and joined MAJU Islamabad Campus in 2007, which was later transformed into Capital University of Science and Technology. He was given such responsibilities as the Head of Mathematics Department and Director Student Affairs in 2012. His research interests are computational fluid dynamics and computational mathematics. Dr. Muhammad Sagheer has published more than fifty research article papers in reputed journals.

Shafqat Hussain completed his $\mathrm{PhD}$ degree from Institute of Applied Mathematics and Numerics, Technische University at Dortmund, Germany. His research area is mainly computational fluid dynamics using Finite Element Method (FEM). He also worked part time as an Assistant Professor at Ruhr-University at Bochum, Bochum, Germany. He joined Mohammad Ali Jinnah University in February 2013 and engaged in various teaching and research activities. Dr. Hussain has supervised many MPhil/PhD projects and published around 40 research articles in the well reputed international journals. 